$\frac{46}{10} 30.9575$ (2)

SANDIA REPORT

SAND95-2139 - UC-404

Unlimited Release

Printed October 1995

\title{
Photocatalytic Semiconductor Clusters for Fuel Production
}

\author{
J. P. Wilcoxon, D. E. Bliss, J. E. Martin, P. P. Newcomer, \\ G. A. Samara, A. Martino, R. L. Williamson
}

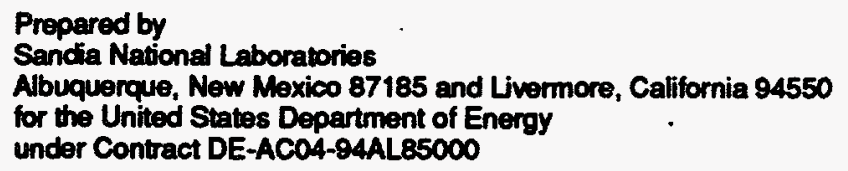

Approved for public release, distribution is unlimited.

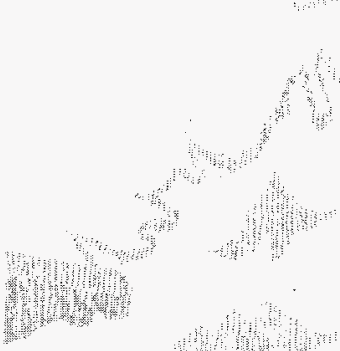


Issued by Sandia National Laboratories, operated for the United States Department of Energy by Sandia Corporation.

NOTICE: This report was prepared as an account of work sponsored by an agency of the United States Government. Neither the United States Government nor any agency thereof, nor any of their employees, nor any of their contractors, subcontractors, or their employees, makes any warranty, express or implied, or assumes any legal liability or responsibility for the accuracy, completeness, or usefulness of any information, apparatus, product, or process disclosed, or represents that its use would not infringe privately owned rights. Reference herein to any specific commercial product, process, or service by trade name, trademark, manufacturer, or otherwise, does not necessarily constitute or imply its endorsement, recommendation, or favoring by the United States Government, any agency thereof or any of their contractors or subcontractors. The views and opinions expressed herein do not necessarily state or reflect those of the United States Government, any agency thereof or any of their contractors.

Printed in the United States of America. This report has been reproduced directly from the best available copy.

Available to DOE and DOE contractors from

Office of Scientific and Technical Information

PO Box 62

Oak Ridge, TN 37831

Prices available from (615) 576-8401, FTS 626-8401

Available to the public from

National Technical Information Service

US Department of Commerce

5285 Port Royal Rd

Springfield, VA 22161

NTIS price codes

Printed copy: A04

Microfiche copy: A01 


\section{DISCLAIMER}

Portions of this document may be illegible in electronic image products. Images are produced from the best available original document. 
SAND95-2139

Distribution

Unlimited Release

Printed October 1995

Category UC-404

\title{
Photocatalytic Semiconductor Clusters for Fuel Production
}

J.P. Wilcoxon, D. E. Bliss, J.E. Martin, P. P. Newcomer, G. A. Samara Nanostructures \& Advanced Materials Chemistry Department

A. Martino

Fuel Science Department

R. L. Williamson

Liquid Metal Processing Department

Sandia National Laboratories

Albuquerque, NM 87185

\begin{abstract}
High quality crystalline, monodisperse nanometer-size semiconductor clusters were successfully grown using an inverse micellar synthesis process and their optical and structural properties were studied. Among the materials studied were PbS, FeS2, $\mathrm{MoS}_{2}, \mathrm{CdS}$ and related compounds. The results demonstrated strong electronic quantum confinement effects and broad tailorability of the bandgaps with decreasing cluster size, features that are important for the potential use of these materials as photocatalysts for solar fuel production and solar detoxification. The highlights of the work are included in an Executive Summary.

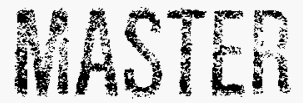




\section{Table of Contents}

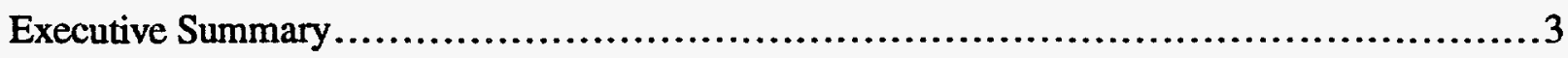

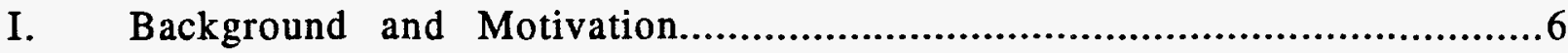

II. Synthesis and Processing of Nanosize Semiconductor

Clusters in Inverse Micelles...................................................... 11

III. Lead Sulfide (PbS) Nanoclusters.............................................................. 14

A. Motivation ............................................................... 14

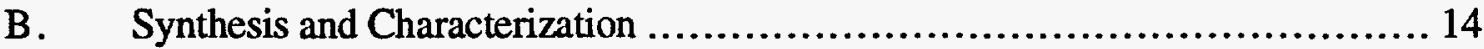

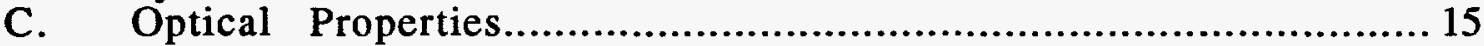

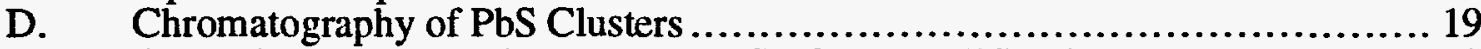

E. Control of Photoluminescence by Surface Modification............................ 23

F. Conclusions .............................................................. 26

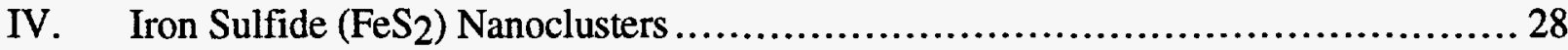

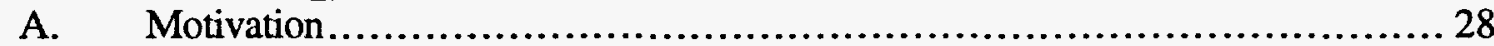

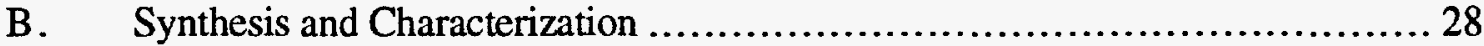

C. Optical Properties: Strong Quantum Confinement Effects.................. 31

D. Surface Modification .................................................... 38

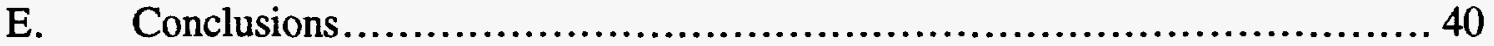

V. Molybdenum Disulfide (MoS2) and Other

Layered Semiconductor Nanoclusters .......................................... 42

A. Motivation ..................................................................... 42

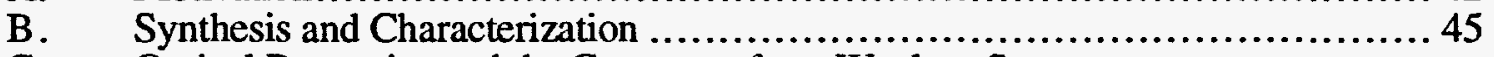

C. Optical Properties and the Crossover from Weak to Strong Quantum Confinement..................................................... 47

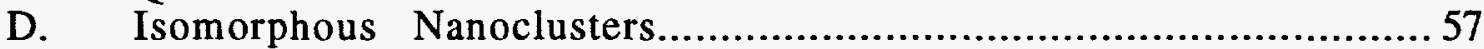

E. Conclusions ............................................................. 60

VI. The Catalyzed Photoreduction of Methyl Orange by

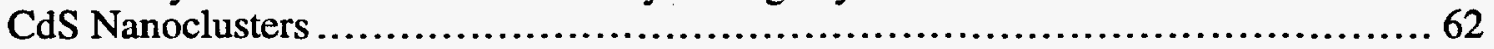

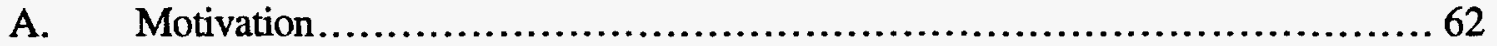

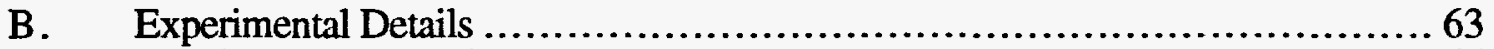

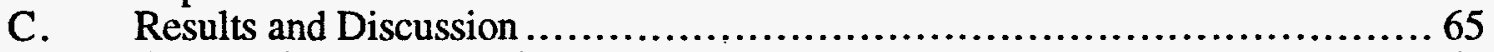

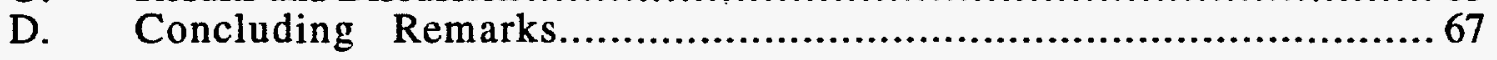

VII. Future Research Directions............................................................ 70

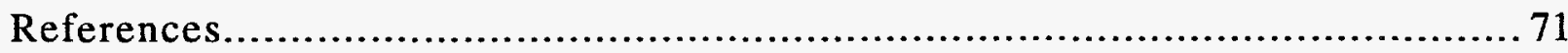

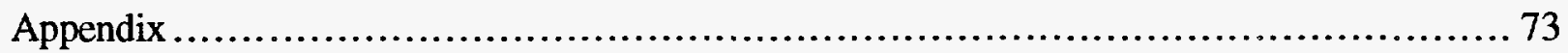




\section{Executive Summary}

The objective of this Laboratory-Directed Research and Development (LDRD) program was to exploit our recently developed inverse micellar synthetic method to produce nanometer-size semiconductor clusters (or nanoclusters) for use as potential photocatalysts for solar fuel production and solar detoxification. In such applications these clusters would absorb light creating electron-hole pairs and thus could catalyze specific chemical reactions such as the decomposition of $\mathrm{H}_{2} \mathrm{O}$ or $\mathrm{H}_{2} \mathrm{~S}$. To be effective as a solar photocatalyst the semiconductor must have a bandgap that is matched to the solar spectrum, and the energies of the valence and conduction level edges must be compatible with the oxidation and reduction potentials for the reactions involved. Additionally, the material must be resistant to photochemical degradation, and the carrier diffusion time to the surface must be shorter than the electron-hole recombination time so that useful interfacial reduction and oxidation may take place.

Semiconductor nanoclusters are in principle ideally suited to meet these requirements. Their small size allows fast carrier diffusion to the surface, and they possess the ability to continuously tune the bandgap (by up to $1 \mathrm{eV}$ or more) by changing cluster size. This is a consequence of the quantum confinement of the electronic charge carriers in small "boxes"; by decreasing the cluster (box) size, we increase the spacings between energy levels leading to an increase in the bandgap and shifts in the band edges.

Highlights of the work are as follows:

- High quality, crystalline, monodisperse nanoclusters of PbS, FeS2, MoS2, CdS and related materials were successfully grown and characterized by electron microscopy, electron diffraction, and other techniques. 
- Strong electronic quantum confinement effects have been observed in the optical absorption and emission properties of these nanoclusters. Their bandgaps, which are typically in the mid to near infrared in the bulk, can be adjusted to the near ultraviolet by reducing the cluster size.

- We have demonstrated that most of the absorbed light energy in $\mathrm{PbS}$ nanoclusters is channeled into a non-radiative process. We observed surfacerelated emission but no bulk recombination, features that bode well for potential applications of these nanoclusters in photocatalysis. Depending on the sign of the change in charge state of the surface recombination sites (or traps), the excited nanoclusters are capable of initiating either reduction (electron trap) or oxidation (hole trap) reactions.

- Results on gold capping of the PbS nanocluster show that surface related states of one material can be excited through states of a different interior material. This effect has extremely important ramifications for photocatalysts since the functions of energy absorption and energy transfer can be separated. Materials for the interior of the nanocluster can be chosen to be efficient absorbers of light energy, while materials on the surface can be chosen to be efficient at transferring energy to reactant molecules.

- Novel well-defined peaks in the optical absorption spectrum of FeS2 have been interpreted in terms of the electronic bandstructure. Most of these peaks have not been observed before in any form of $\mathrm{FeS}_{2}$ and are attributed to the excellent crystal quality and monodispersity of our clusters.

- The bandgap of $\mathrm{FeS}_{2}$ remains indirect down to the smallest cluster size studied $(2 \mathrm{~nm})$; however, the absorbance of the smaller clusters at short wavelengths is considerably larger than that of larger clusters.

- The ability to adjust the absorption spectra of $\mathrm{MoS}_{2}$ and its isomorphs to match the solar spectrum along with the known high resistance of these materials against photocorrosion make them prime candidates for solar photocatalysis. The corrosion resistance is related to the antibonding nature of the state responsible for the optical transition at the top of the valence band.

- Our results on $\mathrm{MoS}_{2}$ nanoclusters provide a demonstration of the crossover from band-like (solid) to molecular-like spectra as the size of the nanoclusters becomes smaller than the size of the exciton in the bulk.

- We demonstrated the photoreduction of methyl orange catalyzed by CdS nanoclusters grown in inverse micelles. However, the reaction rate in inverse 
micelles in octane was slower than the analogous reaction using CdS powders dispersed in water. In retrospect, such a reaction using a water-soluble molecule such as methyl orange would be much more efficient if our CdS nanoclusters were transferred to an aqueous medium. However, we anticipate that other reactions using an oil soluble reactant might work quite effectively. Some photo-decomposition of the CdS nanoclusters was evident on the time scale of the experiments. We showed that this degradation mechanism can be controlled by coating the clusters with a thin metal layer, or by using ions which can act as hole traps at the surface (e.g. HS-)

Finally, this LDRD program has made significant progress toward the ultimate goal of using nanoclusters as efficient photocatalysts. The specific direction of our research was guided by a practical consideration-the need for inexpensive, disposable photocatalysts. This consideration led us to emphasize sulfide nanoclusters. Much more work is needed to develop these materials and apply them for such important reactions as the decomposition of $\mathrm{H}_{2} \mathrm{O}$ and $\mathrm{H}_{2} \mathrm{~S}$ and the solar detoxification of chlorinated hydrocarbons in water. 


\section{Background and Motivation}

It has long been recognized that the success of a material as a catalyst is critically dependent on the specific surface area[1] (i.e. the surface area per gram). In order to have large surface areas available for chemical reactions, the conventional approach is to deposit a metal onto a substrate which has an inherently large surface area (e.g. highly divided alumina or titania). Such heterogeneous catalysts play a central role in oil refining and chemical production. Alternatively, the metal might be dispersed at the molecular level, forming a homogenous catalyst. In this latter case the metal lacks the translation symmetry characteristic of the bulk, and these catalysts are truly molecular in nature.

In the work presented in this report, we describe a new class of unsupported semiconductor materials with physical properties in between those of the bulk and the molecular level. These materials consist of clusters from a few dozen to thousands of atoms which are completely dispersed in an organic oil phase. They are stabilized against aggregation by the presence of surfactants, and are potentially capable of functioning as catalysts for a variety of photocatalytic reactions.

We have recently developed a unique synthesis process, based on the use of inverse micelles as reaction vessels, that is capable of producing monodisperse clusters from a variety of different materials down to $\sim 1 \mathrm{~nm}$ in size [2]. This

process provided the basis for this Laboratory-Directed Research and Development (LDRD) program. 
The overall objective of this LDRD program was to capitalize on our inverse micellar approach to produce nanometer-size semiconductor clusters (nanoclusters for short) as potential photocatalysts for solar fuel production and solar detoxification. In such an application these clusters would absorb light creating electron-hole pairs and thereby catalyzing specific chemical reactions, such as the decomposition of $\mathrm{H}_{2} \mathrm{O}$ and $\mathrm{H}_{2} \mathrm{~S}$, when dispersed in solution. To be effective as a solar photocatalyst the semiconductor must have a bandgap that is matched to the solar spectrum, and the energies of the valence and conduction band edges must be compatible with the oxidation and reduction potentials for the reactions involved. Additionally, the material must be resistant to photochemical degradation, and the carrier diffusion time to the surface (where the chemistry takes place) must be shorter than the electron-hole recombination time.

Because of their small size, nanoclusters are in principle ideal for the latter requirement. To appreciate this feature, consider the competing recombination and charge diffusion processes in $\mathrm{TiO}_{2}$ clusters. The average transit time to the surface, $t=R^{2} / p^{2} D$, increases with the square of the cluster radius $R$ and depends inversely on the electron diffusion constant $D$. For a radius of $10 \mathrm{~nm}$, which we can readily achieve using our micellar microreactors, and an electron mobility of $0.5 \mathrm{~cm}^{2} / \mathrm{Vs}$, the transit time is $10 \mathrm{ps}$. Under laser irradiation a majority carrier density of $10^{19} \mathrm{~cm}^{-3}$ can be achieved, which gives a recombination time of 100 ps. Thus, even under strong irradiation recombination is negligible in nanoscale clusters. However, in commercial $\mathrm{TiO}_{2}$ powders with a typical radius of 1000 $\mathrm{nm}$, the transit time from the bulk to the surface would be $100 \mathrm{~ns}$ and charge recombination would dominate. This illustrates the principal advantage of semiconductor nanoclusters (with $\mathrm{R} \leq 10 \mathrm{~nm}$ ) in artificial photosynthesis. 
Because of its chemical stability in photoelectrochemical environments, titanium dioxide $\left(\mathrm{TiO}_{2}\right)$ powder has been extensively studied as a model system. Unfortunately, $\mathrm{TiO}_{2}$ has too wide of a bandgap ( $>3 \mathrm{eV}$ ) and only absorbs light shorter than $400 \mathrm{~nm}$, which constitutes less than $10 \%$ of the solar radiation. A variety of other semiconductors whose bandgaps are better matched to the solar spectrum (e.g., II-VI semiconductors such as $\mathrm{CdS}$ ) have been studied; however, a major disadvantage of these materials in bulk or powder forms is their rapid photoelectrochemical degradation. Other bulk or powder materials, in particular MoS2, have been found to be highly stable [3]; however, their bandgaps are in the near infrared, i.e., too small.

One of the most attractive properties of semiconductor nanoclusters is the ability to continuously tune the bandgap (by up to $1 \mathrm{eV}$ or more) by changing cluster size. This is a consequence of the quantum confinement of the electronic charge carriers in small "boxes"; by decreasing the cluster (box) size, we increase the spacings between energy levels leading to an increase in bandgap. Thus, while bulk or powder $\mathrm{MoS}_{2}$ is not very attractive as a solar photocatalyst, the ability to tailor its bandgap in nanocluster form is a very attractive possibility which constituted a part of the motivation for our work.

The specific objectives of this LDRD program were to develop new semiconductor nanoclusters with tailorable bandgaps using inverse micellar synthesis, to characterize their size and crystal structure, to measure their optical properties, to demonstrate the ability to coat their surfaces, e.g. by metals, as a first step toward the ultimate development of photochemical half cells, and to demonstrate their ability to photocatalyze chemical reactions. As will be shown in this report, considerable progress was made toward these ends. 
From the outset, the specific directions of the program were guided by practical considerations. The intended solar photochemical applications for fuel production (e.g., the decomposition of $\mathrm{H}_{2} \mathrm{O}$ and $\mathrm{H}_{2} \mathrm{~S}$ ) and solar detoxification (e.g., the destruction of chlorinated hydrocarbons in water) require the capability to produce large quantities of inexpensive semiconductor nanoclusters which most likely will not be recovered for reuse. Being a solution process, our inverse micellar synthesis approach is amenable to scale-up to essentially any desired size. Additionally, we concentrated on suitable materials which are common and could be produced inexpensively in cluster form. Consequently, we emphasized metal sulfides, and more specifically those of molybdenum $\left(\mathrm{MoS}_{2}\right)$, iron $\left(\mathrm{FeS}_{2}\right)$, lead $(\mathrm{PbS})$ and several others including cadmium (CdS) which in bulk or powder form has been a model photocatalyst despite its photochemical degradation.

Our chosen materials offer some side benefits. $\mathrm{FeS}_{2}$ and $\mathrm{MoS}_{2}$ materials in bulk form have significant technological applications. For example, cubic iron sulfide is considered to be the most important mineral present in coal formations and likely plays a critical catalytic role in hydrogenolysis of coal.[4] It has also been suggested as an inexpensive material for photovoltaic applications.[5] $\mathrm{MoS}_{2}$ is the most widely used catalyst for the removal of sulfur from crude oil and coal and, due to its graphite-like two-dimensional structure, is widely used in lubrication applications (e.g. axle grease). The availability of these materials in cluster form can be expected to enhance these applications as well as lead to new possibilities.

The report is organized as follows. Section II provides a brief description of the inverse micellar synthesis process. Section III deals with lead sulfide ( $\mathrm{PbS})$ nanoclusters. Although $\mathrm{PbS}$ is not to our knowledge known to be an effective catalyst in bulk or powder form, we selected it as a model system because of its 
simple crystal and electronic structures and because its very small bulk bandgap affords a broad range of bandgap tailorability by decreasing cluster size. As will be shown, much has been learned from this model system about carrier absorption and emission of nanoclusters and about the role of surface modification in controlling these properties.

Sections IV and $\mathrm{V}$ discuss iron sulfide $\left(\mathrm{FeS}_{2}\right)$ and molybdenum sulfide $\left(\mathrm{MoS}_{2}\right)$ and ismorphous layered semiconductor nanoclusters, respectively. Much was learned about the controlled synthesis and properties of these nanoclusters which are very attractive candidates for photocatalysis as well as catalysis. Section VI discusses the photoreduction of methyl orange catalyzed by CdS nanoclusters. Section VII provides concluding remarks and thoughts about future directions for this research. 


\section{Synthesis and Processing of Nanosize Semiconductor Clusters In Inverse Micelles}

It is well known that size control during colloidal growth requires the distinct separation of nucleation from growth. If nucleation occurs continuously, in either space or time, a highly disperse (power-law distribution) of cluster sizes will inevitably occur. Such processes are typical of growth in a continuous medium whether gas or liquid. However, if confinement of the reactant precursors can be achieved to limit growth to a restricted region of space, a narrow size distribution of clusters will occur. Examples include cluster growth in mineral zeolites and emulsion polymerization to form latexes (the latter process produces the most highly monodisperse objects yet grown).

In our synthetic method[6] we achieve this desired spatial restriction by the confinement of the reactants (i.e. metal salts such as $\mathrm{Li}_{2} \mathrm{~S}$ or $\left.\mathrm{Fe}\left(\mathrm{BF}_{4}\right)_{2}\right)$ in the hydrophilic interior of surfactant aggregates called inverse micelles. This is depicted in Figure 1. Surfactant molecules, the dipoles of the chemical world, have versatile solubility characteristics (i.e. they like water, but they're also comfortable in oil). However, in either case they usually form segregated droplet-like aggregates called micelles whose dimensions are typically $1-10 \mathrm{~nm}$. The modifier "inverse" refers to the negative curvature between the surfactant-oil interface (the oil is the continuous medium). Nucleation only in the micelle interior is guaranteed by the total lack of solubility of the charged metal ions in the low dielectric constant inert oils used as the continuous media. The micelles essentially function as "nano-glassware" to grow the clusters. Since there are roughly $10^{18}$ such micelles per $\mathrm{cm}^{3}$, a lot of identical clusters can be grown. 
The size of these molecular reactors is determined by the chemical structure of the surfactants and their interactions with the oil.

Metal sulfide clusters were formed by mixing two precursor micelle solutions (e.g. $\mathrm{Fe}(\mathrm{ClO})_{2}$ and $\mathrm{Na}_{2} \mathrm{~S}$ ) which would ordinarily form a bulk precipitate of $\mathrm{FeS}_{2}$. Metal oxide clusters (e.g. TiO2) were formed by forced hydrolysis of $\mathrm{TiCl}_{4}$ using a base such as $\mathrm{NaOH}$. Also, a seed population of small inexpensive semiconductor clusters (e.g. FeS2) was first prepared as outlined above and subsequently coated with another more expensive but active catalyst, (e.g. Pd). This allowed us to protect the clusters from chemical oxidation, a major goal of this program.

Of course, after formation of the clusters it's time to separate reactants from products (the clusters). Reaction byproducts were removed at the molecular level by high pressure liquid chromatography (HPLC), which separates products (clusters) from reactants (surfactants, oils, and inorganic salts). This "cleaning" process is quite important to allow measurement of the inherent optical features of the clusters, especially features in the deep UV. The development of these unique processing methods is one of the most significant and unanticipated results of this LDRD and opens up a host of new possibilities for better materials which has already led to increased scientific understanding and potential technological application of these new materials. It also allows us to modify the cluster surface and then disperse the modified clusters in a solvent which differs significantly from that used for synthesis (e.g. changing from octane to water). 


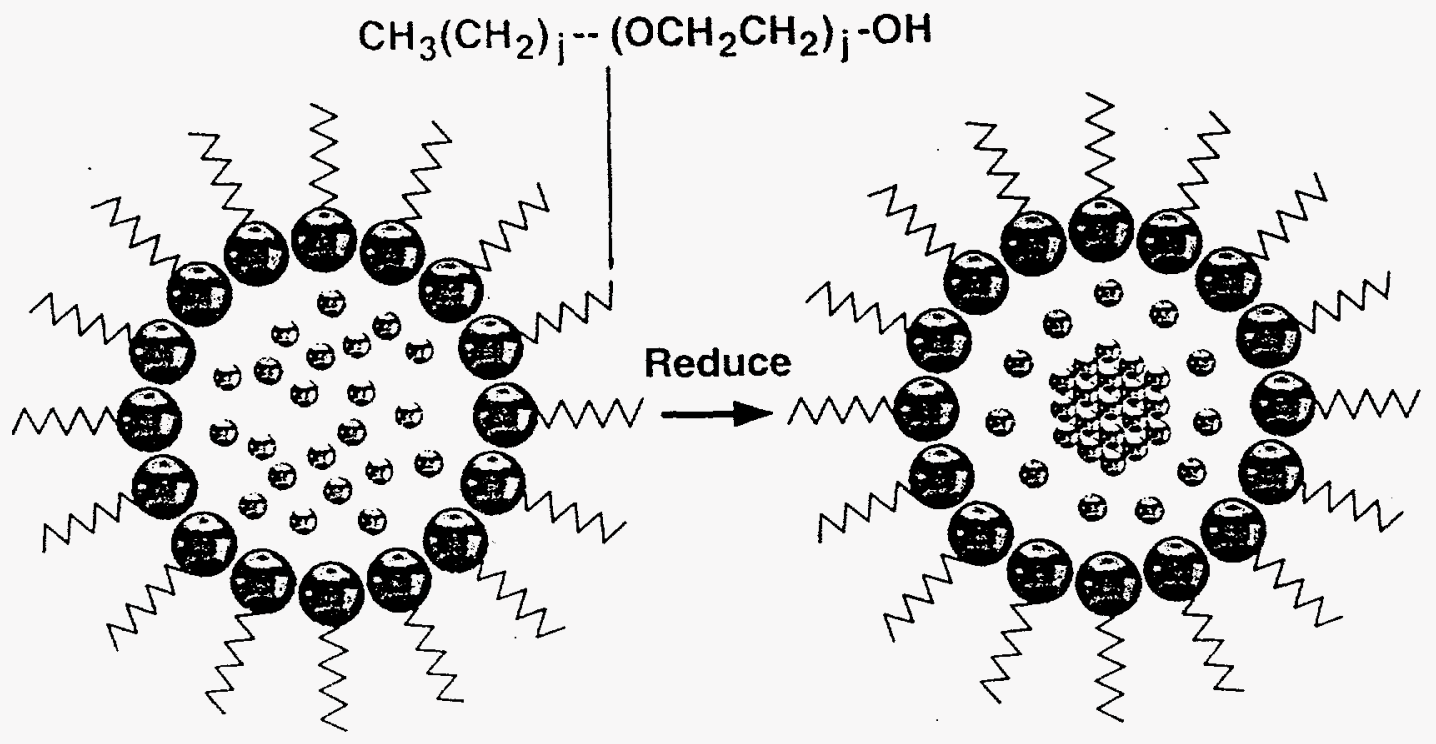

Figure 1. Schematic representation of the synthesis of nanometer-size clusters inside inverse micellar cages. The interiors of the cages are typically $1-5 \mathrm{~nm}$ in size. The hydrocarbon tails of the surfactant molecules form an interface with the continuous nonpolar medium. 


\section{Lead Sulfide (PbS) Nanoclusters.}

\section{A. Motivation}

$\mathrm{PbS}$ is a model material system to study the effects of size confinement and surface modification in nanoclusters. It possesses a simple crystal structure (cubic rock salt) and a simple electronic structure. $\mathrm{PbS}$ has a small direct bandgap at the $L$ point of the Brillouin zone which is well isolated from other bands, simplifying the interpretation of optical absorption spectra. The bulk material properties are well understood. The small effective mass and large dielectric constant yield a large excitonic Bohr radius, $\mathrm{r}_{\mathrm{B}} \sim 17 \mathrm{~nm}$. PbS nanoclusters smaller than this exciton size are easily synthesized in inverse micelles, indicating that strong electronic confinement effects should be readily achieved.

\section{B. Synthesis and Characterization}

A series of $\mathrm{PbS}$ nanoclusters were synthesized using either $\mathrm{Pb}\left(\mathrm{NO}_{3}\right)_{2}$ or $\mathrm{Pb}\left(\mathrm{ClO}_{4}\right) 2$ salt concentrations of $1 \times 10^{-4}, 2 \times 10^{-4}, 5 \times 10^{-4}, 1 \times 10^{-3} \mathrm{M}$ and $2 \times 10^{-3}$ $\mathrm{M}$ and stoichiometry ratios $\mathrm{Pb}: \mathrm{S}$ of 2:1, 1:1, 1:2. Appropriate amounts of sulfiding agent (either $\mathrm{Li}_{2} \mathrm{~S}$ or $\mathrm{H}_{2} \mathrm{~S}$ ) were then delivered in another inverse micelle solution with rapid stirring to mix the components. Reaction was instantaneous in most cases. Size and structure determinations were done by transmission electron microscopy (TEM). Selected area diffraction and lattice fringe images of alternating planes of $\mathrm{Pb}$ and $\mathrm{S}$ atoms confirm that these $\mathrm{PbS}$ nanocrystals have the bulk rock salt structure. This shows that single crystal nanoclusters can be formed by room temperature synthesis. The diameters of the particles were measured from TEM micrographs. The general trend is that the particle size increases with reagent concentration in the range of $2-5 \mathrm{~nm}$. 


\section{Optical Properties}

Figure 2 shows the optical absorbance of the PbS clusters synthesized at 1:1 stoichiometry. Samples \#80 and \#81 have an absorption peak at $4.37 \mathrm{eV}(284$ $\mathrm{nm})$. As the concentration is increased a background absorption develops and the relative intensity of the $284 \mathrm{~nm}$ peak decreases. The fact that the peak position does not depend on cluster size suggests that it is a molecular level and not an excitonic feature. TEM studies indicate the average cluster diameter for these samples shown in Figure 2 were $2.4 \mathrm{~nm}$ (\#80), 3.0 (\#81), $3.6 \mathrm{~nm}(\# 82), 6.5$ (\#83), and $7.9 \mathrm{~nm}(\# 84)$.

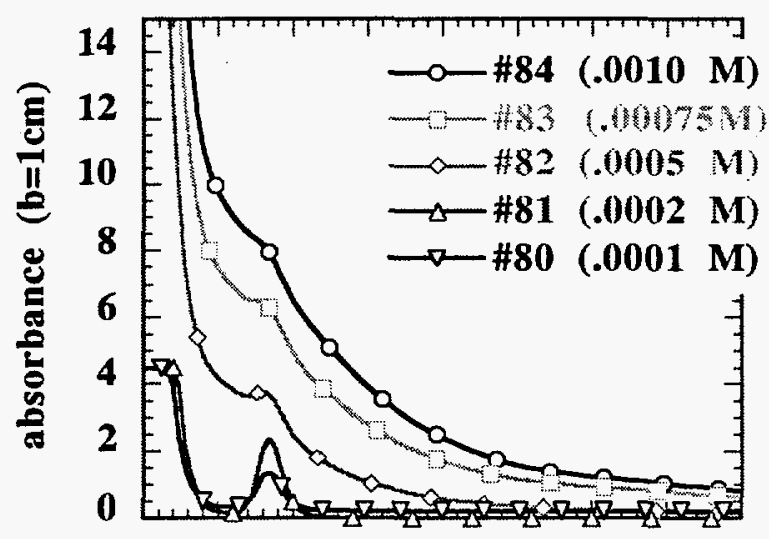

200250300350400450500550600 wavelength $(\mathrm{nm})$

Figure 2. Optical absorbance of $\mathrm{PbS}$ clusters for a range of $\mathrm{Pb}$ concentrations.

Figure 3 compares the absorption spectrum of $3 \mathrm{~nm}$ clusters to that of matrix isolated diatomic $\mathrm{PbS}$ monomers [7]. The strongest $\mathrm{UV}$ absorption feature of the diatomic $\mathrm{PbS}$ molecules matches the peak of our nanoclusters in energy. This state is labeled $\mathrm{E}$ and has been tentatively identified as the transition to the excited singlet molecular orbital $\pi^{3} \sigma^{2} \pi,{ }^{1} \Sigma^{+}$[8]. The $\mathrm{E}$ transition has no vibrational structure and is thought to be a pre-dissociative excitation since the 
dissociative energy, $4.7 \mathrm{eV}$, for $\mathrm{PbS}$ is greater than the peak photon energy of hv $=4.37 \mathrm{eV}(284 \mathrm{~nm})$.

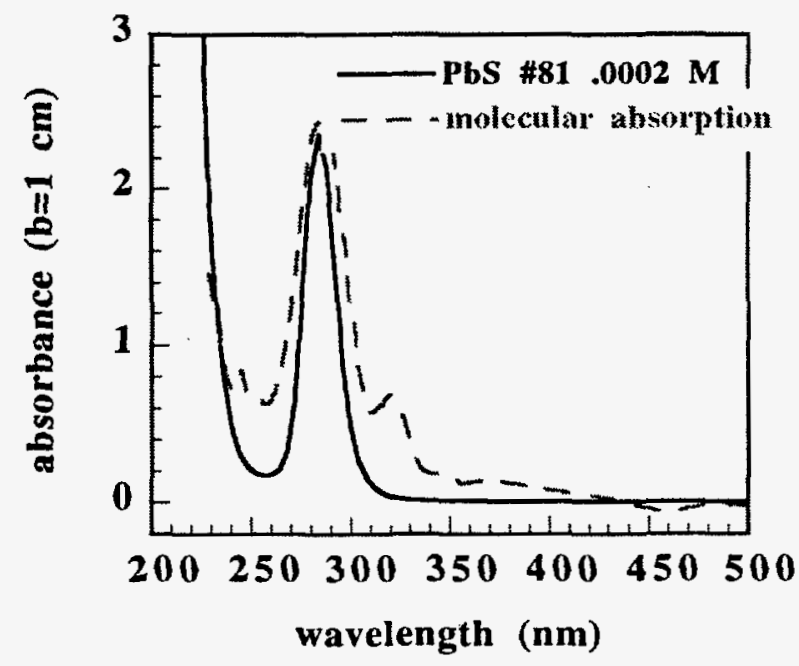

Figure 3. Comparison of absorption between our nanoclusters (sample \#81, diameter $=3.0 \mathrm{~nm}$ ) and matrix isolated diatomic $\mathrm{PbS}$ molecules [7].

The photoluminescence emission (PL) and excitation (PLE) spectra are shown in Figure 4. A strong emission band occurs at $520 \mathrm{~nm}$. The PLE spectrum for the $520 \mathrm{~nm}$ emission exhibits two peaks at 270 and $300 \mathrm{~nm}$. Since the emission occurs at a much lower energy than either the excitation or absorption features, it is interpreted to result from a surface state or defect. 


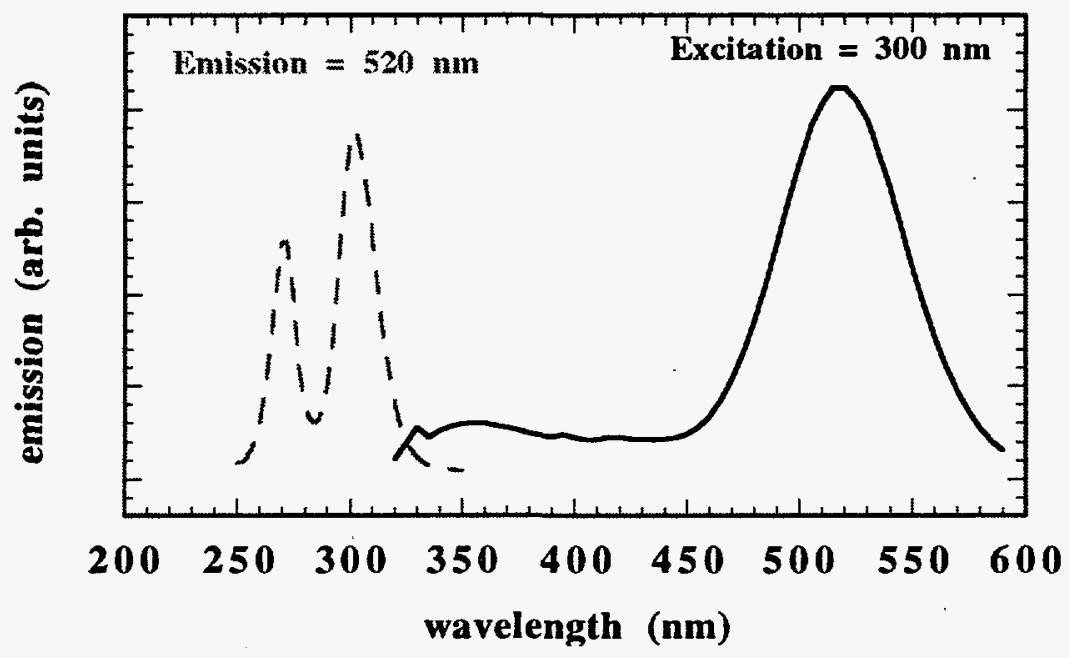

Figure 4. PL emission and excitation spectra of $\mathrm{PbS} \# 81$.

Figure 5 shows a comparison of typical PLE and absorption spectra. The fact that the absorption peak lines up with the valley between the two excitation peaks suggests that it is cutting a hole in the excitation band. Most of the absorbed light energy is channeled into a non-radiative process. This bodes well for applications of this nanosize material in photocatalytic applications. The amount of pump beam intensity lost to this nonradiative absorption can be easily calculated. The geometry of our PL setup is such that the pump beam passes through a $1 \mathrm{~cm}$ cell and the emitted light is collected at right angles over the center $0.5 \mathrm{~cm}$ of the cell. Equation 1 gives the corrected PL emission, $\varepsilon$, as

$$
\mathcal{E}=\frac{E \ln (10) A}{\left(10^{-A / 4}-10^{-3 A / 4}\right)},
$$

where $\varepsilon$ is the measured PLE intensity as a function of wavelength and $A$ is the measured absorbance of the $1 \mathrm{~cm}$ cell. A corrected PLE spectrum is shown in Figure 5. The simple geometrical correction of Eq. 1 indeed yields a single Gaussian peak for the excitation band. 


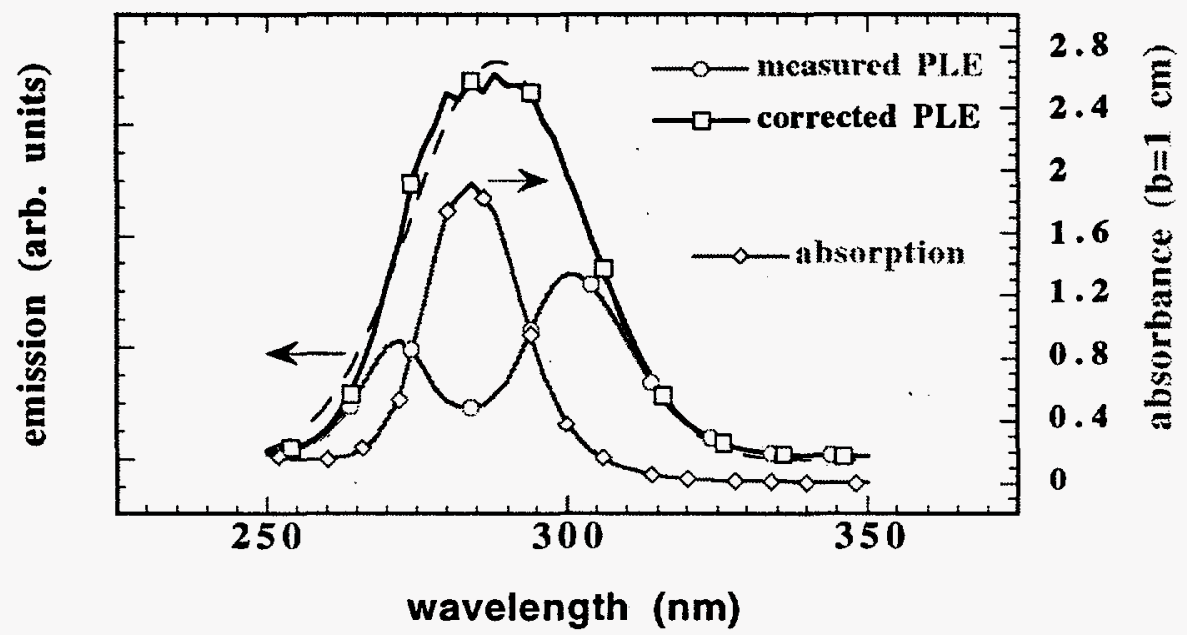

Figure 5. PLE and absorbance spectra of PbS \#94 (1:2, 2x10-4 M). The curve with square symbols represents the PLE spectrum corrected by Eq. 1 for the absorption. The dashed line is a Gaussian fit to the corrected data.

The corrected PLE band has a full-width-half-maximum linewidth of 47 $\mathrm{nm}$ which is approximately double the absorption linewidth of $27 \mathrm{~nm}$. The corrected PLE band is also red shifted $0.1 \mathrm{eV}(6 \mathrm{~nm})$ relative to the absorption peak. The intensity of the corrected PLE peak drops precipitously with increasing cluster size.

Our interpretation of the absorption and PLE peaks is that they both are excitations of levels derived from the $E$ electronic state of diatomic $\mathrm{PbS}$. The difference is that the absorption peak is due to $\mathrm{PbS}$ molecules on the surface and the PLE peak is due to molecules in the interior of the cluster. A strong PLE peak is only seen in the smallest clusters, $\sim 2.5 \mathrm{~nm}$ in diameter. This would correspond to a cluster containing approximately $25 \mathrm{PbS}$ molecules in the interior. In terms of a qualitative tight binding model, the interactions between the coordinated molecules in the interior broadens the molecular level. Hence the PLE band is the broader band. One might suspect that the excited electronic state of the $\mathrm{PbS}$ molecule on the surface should also be shifted or broadened by the 
presence of the nanocluster, but this is not observed. Earlier it was pointed out that the $E$ excitation is a pre-dissociative excitation of diatomic $\mathrm{PbS}$ (no vibrational structure exists because there is no restoring force). A dissociative excitation on the surface means the excited atoms leave the cluster. Therefore, the position of the absorption line is not shifted since the excited state cannot interact with the nanocluster.

The dissociative nature of the excitation also explains why no related PL is observed. A dissociative excitation is self trapping in the sense that the excitation energy is carried away by the fleeing molecule. Thus, the energy of this excitation is lost from the nanocluster. On the other hand, excitations of the interior molecules can couple to the cluster surface via delocalization and trapping, or more likely, a long range dipole-dipole interaction. The unknown surface state at $520 \mathrm{~nm}$ could be stimulated via this excitation. The fact that we fail to observe bulk recombination and only see the surface-related emission supports the concept that $\mathrm{PbS}$ nanoclusters should make good photocatalysts. Depending on the sign of the change in charge state of the surface trap, the excited nanocluster is capable of initiating either reduction (electron traps) or oxidation (hole traps) reactions.

\section{Chromatography of $\mathrm{PbS}$ Nanoclusters.}

The identification of the sharp absorbance at $\sim 280 \mathrm{~nm}$ with that seen in molecular $\mathrm{PbS}$ suggests the possibility that this absorbance might arise from true molecular-size $\mathrm{PbS}$ mixed in with the $3.0 \mathrm{~nm}$ size $\mathrm{PbS}$ clusters. Figure 6 shows a lattice fringe image of a $3 \mathrm{~nm} \mathrm{PbS}$ cluster; however $\mathrm{PbS}$ molecules would be impossible to observe even by HRTEM. A bimodal size distribution of $\mathrm{PbS}$ clusters would easily be observed by chromatography. Two population of 
clusters, nanosize and molecular size would show two separate peaks in a chromatogram.

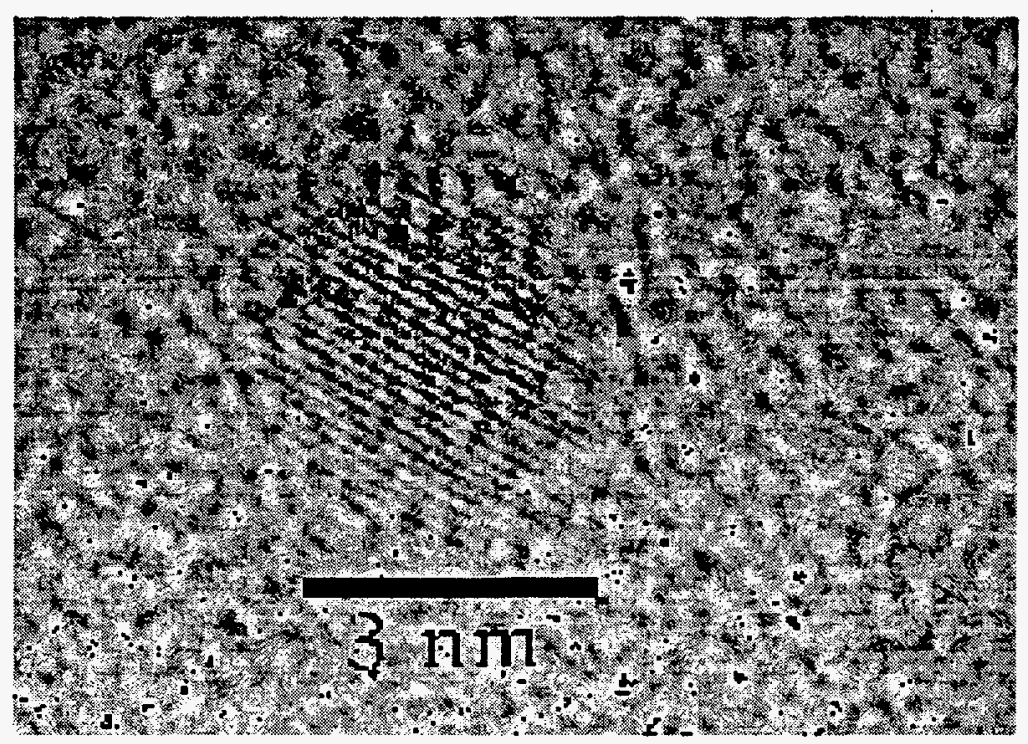

Figure 6. Lattice fringe image of a $3 \mathrm{~nm} \mathrm{PbS}$ cluster.

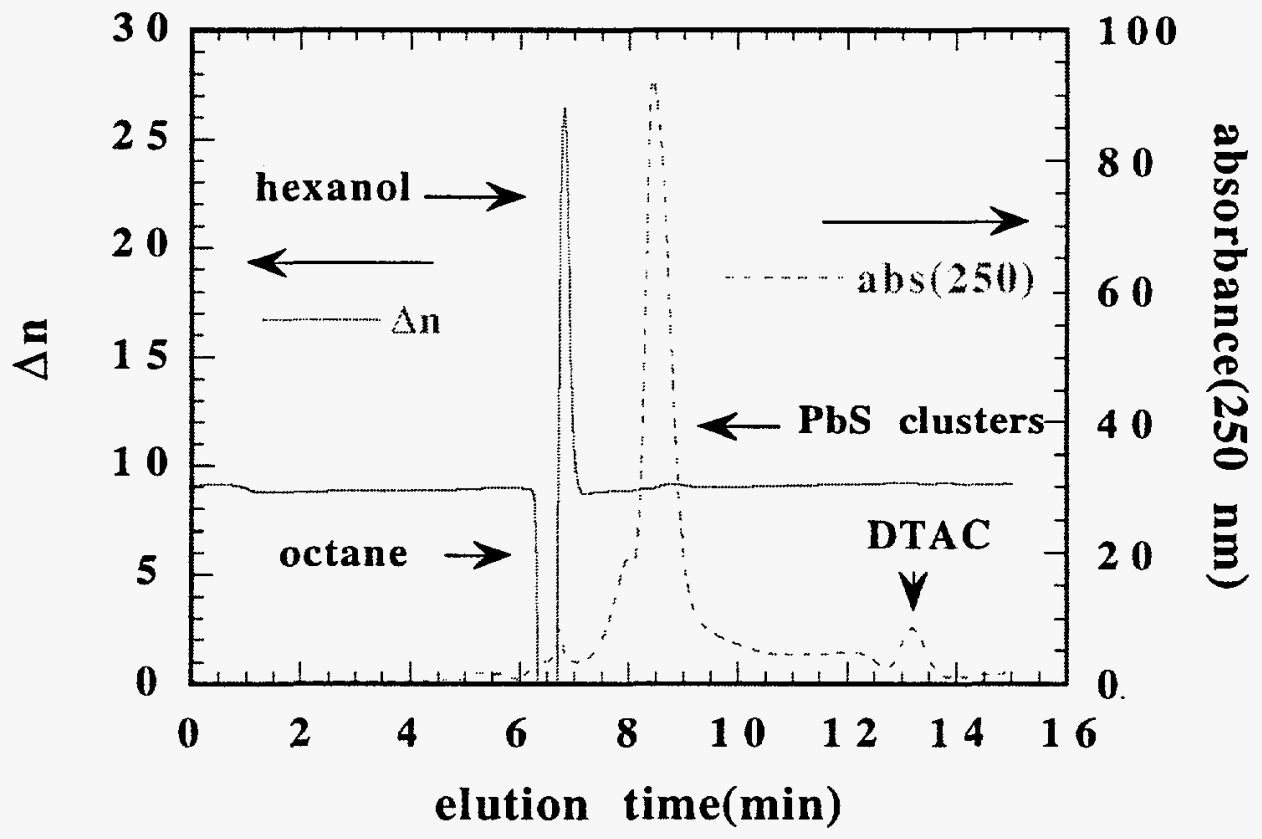

Figure 7. Chromatogram of $\mathrm{PbS}$ in an DTAC/hexonal/octane inverse micelle system. Differential refractive index $\Delta \mathrm{n}$ and the absorbance at $250 \mathrm{~nm}$ are plotted vs. the elution time. Each response is monitored by a separate detector. 
We used liquid chromatography to purify the $\mathrm{PbS}$ cluster solution whose spectral absorbance and emission characteristics were shown previously in Figures 3 and 4. This sample was synthesized in an inverse micelle system containing the surfactant DTAC, hexanol, and octane. Each of these components can be identified and shown to be separated from the clusters as revealed in the chromatogram of Figure 7.

In this separation a porous silica-size exclusion column with $300 \AA$ pores was used and tetrahydrofuran (thf) was the mobile medium. Since octane has a lower refractive index than thf while hexanol has a higher one, the negative and then positive refractive index changes show the separation of both hexanol and octane from the clusters. The cluster elution peak was identified by examination of the on-line PDA output spectrum shown in Figure 8. This spectrum shows the molecular-like feature observed in the parent, unpurified sample of Figure 3. The PL from all components (not shown) was also monitored and shows a response only at the position of the PbS cluster peak demonstrating that there is only one component, and the observed PL is not occurring from an independent, molecular-size $\mathrm{PbS}$ population. 


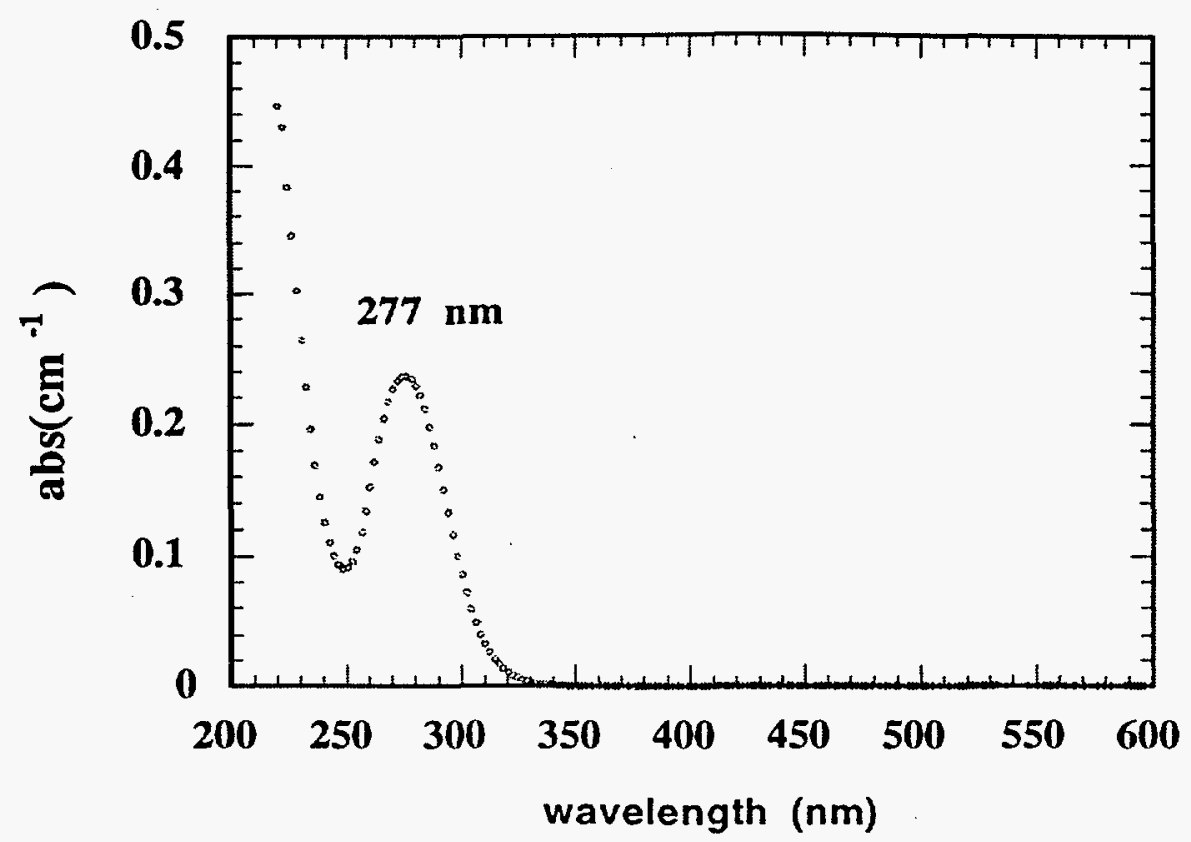

Figure 8. Absorption spectrum at the apex of the $\mathrm{PbS}$ cluster elution peak at 8.5 minutes shown in the chromatogram of Figure 7.

The ability to monitor the purified cluster peak on-line during the chromatography allowed us to investigate the inherent photoluminescence of the purified PbS clusters. The results are shown in Figure 9 and may be compared to the unpurified parent solution shown in Figure 4. Though the emission spectrum of the purified solution is quite similar to the parent solution, it is clear that reaction byproducts are distorting the true excitation spectrum. In particular, excitation at both 420 and $300 \mathrm{~nm}$ (not shown) yields an emission peak at $520 \mathrm{~nm}$ in the purified sample. For reasons we do not yet fully understand, the strong PLE band at $420 \mathrm{~nm}$ is absent from the parent solution of Figure 4 . 


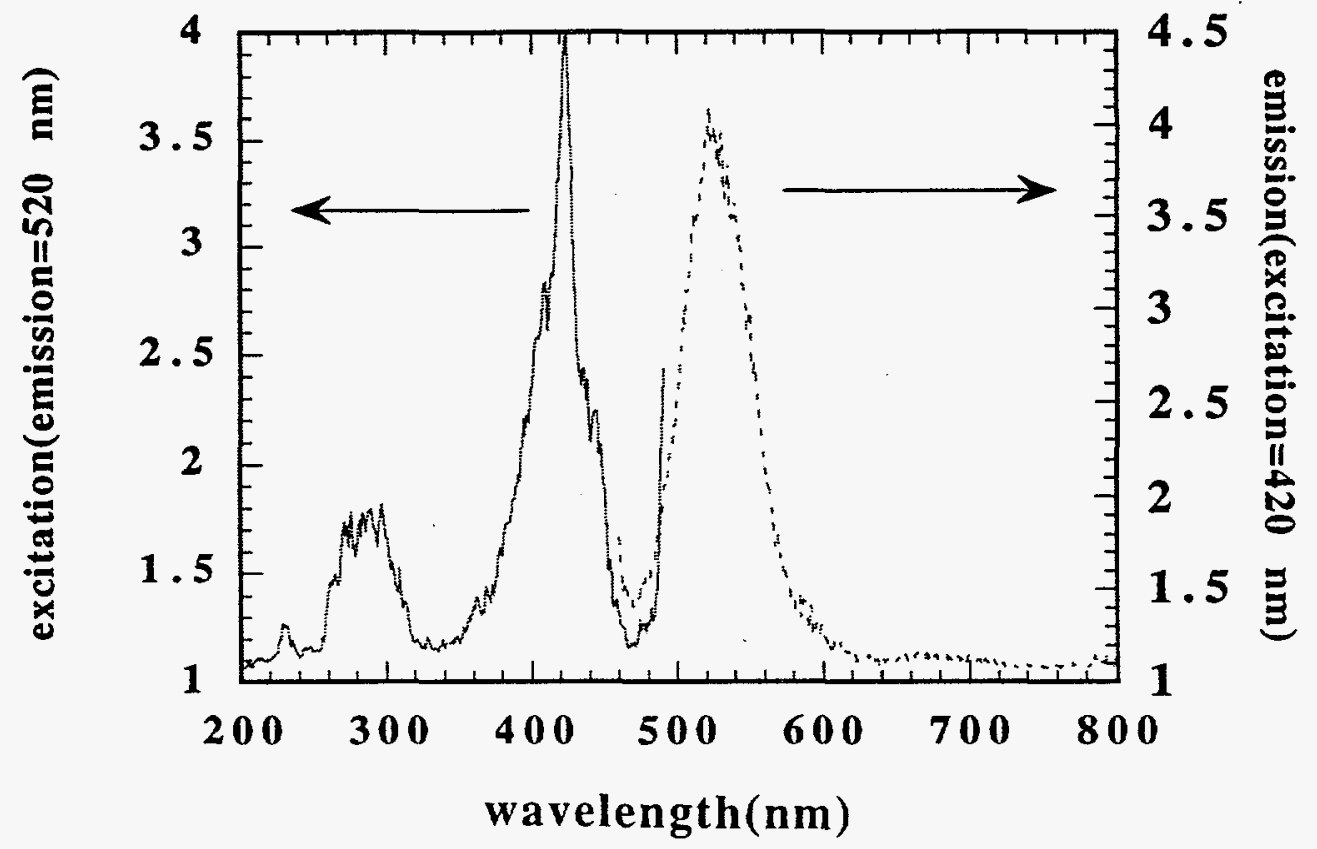

Figure 9. Photoluminescence and excitation spectra of the 8.4 minute $\mathrm{PbS}$ nanocluster elution peak of Figure 7.

\section{E. Control of Photoluminescence by Surface Modification}

We speculated at the start of this program that it should be possible to alter the chemistry of the cluster surface so as to enhance the desired trapping of carriers and reduce undesired photoluminescence. For example, one would expect that the presence of excess $\mathrm{Pb}^{+2}$ ions at the surface would enhance electron trapping while excess $S^{-2}$ would similarly trap holes and reduce recombination. We synthesized identically sized $\mathrm{PbS}$ with $2: 1,1: 1$, and $1: 2 \mathrm{~Pb}: \mathrm{S}$ stoichiometry to test this hypothesis. The results are shown in Figure 10. It appears that recombination is primarily occurring at hole traps since the photoluminescence is considerably reduced by the presence of excess $S^{-2}$ at the cluster surface. Surprisingly, the presence of excess $\mathrm{Pb}+2$ at the surface actually increases the PL compared to the neutral clusters. This illustrates the complexities involved in fine tuning the electronic properties of nanoclusters. 


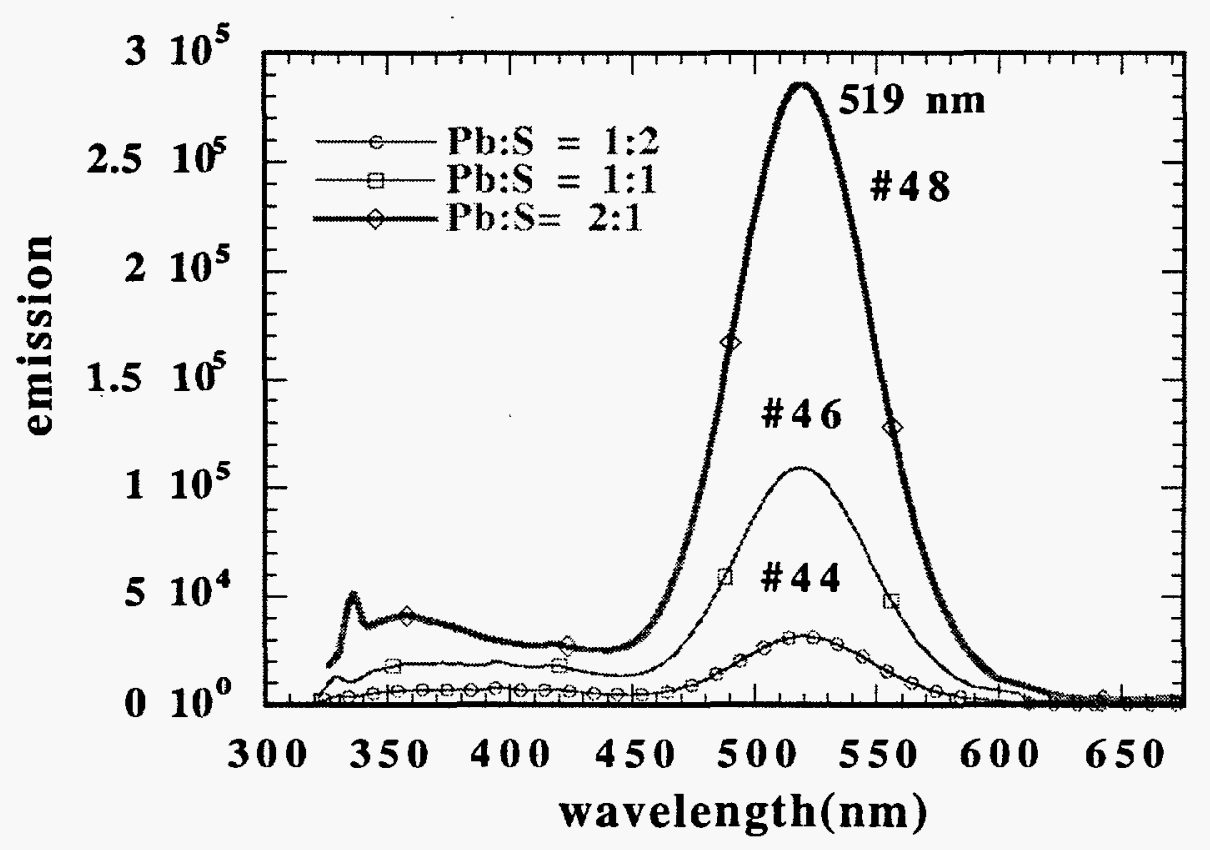

Figure 10. Effect of excess $\mathrm{Pb}^{+2}$ or $\mathrm{S}^{-2}$ ions on observed photoemission from $3.0 \mathrm{~nm}$ PbS clusters.

As a further perturbation of the surface, the $\mathrm{PbS}$ clusters were coated with Au. A concentration of $5 \times 10^{-5} \mathrm{M}$ Au ions was added to a $2 \times 10^{-4} \mathrm{M}$ solution of $\mathrm{PbS}$ nanoclusters and reduced. Given the $3.0 \mathrm{~nm}$ diameter of the clusters, this is just under a monolayer of Au. The effect of the Au on the PL spectrum is quite dramatic. Figure 11 shows that the emission at $520 \mathrm{~nm}$ is completely killed off and a new emission occurs at $350 \mathrm{~nm}$. This indicates that the $520 \mathrm{~nm}$ emission is indeed a surface emission of the PbS cluster. The new $350 \mathrm{~nm}$ emission is similar to that seen from the surfaces of Au clusters in surfactant solutions but with a distinct difference: The PLE spectrum is not the same as for pure Au clusters in solution. Rather, it is the familiar PLE spectrum of PbS. Thus Au-related levels on the surface can be stimulated through the excitation of PbS-related levels in the interior of the cluster. Figure 12 shows that the absorption peak of the Au- 
coated $\mathrm{PbS}$ nanoclusters is reduced in intensity and blue shifted by $\sim 0.1 \mathrm{eV}$. This effect is consistent with the interpretation of the peak as due to $\mathrm{PbS}$ molecules on the surface.

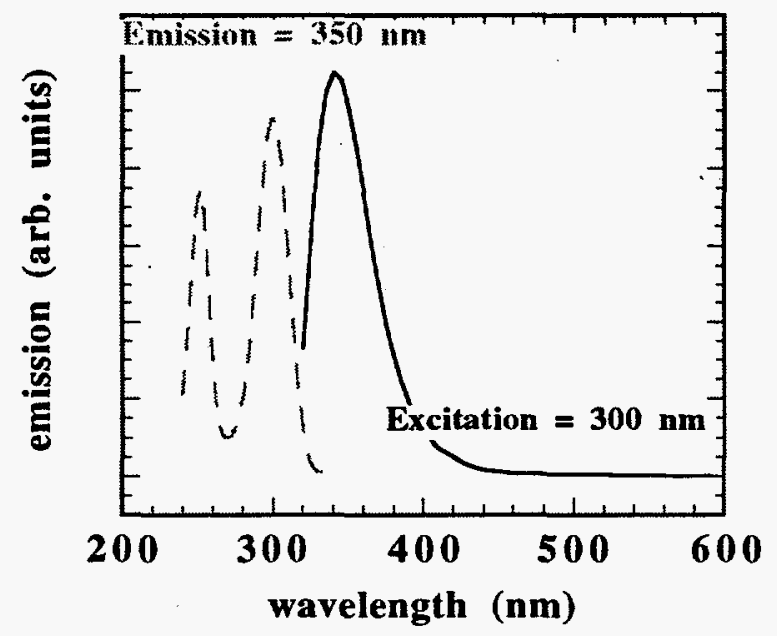

Figure 11. PL and PLE of gold-coated PbS clusters. Refer to Figure 4 for the PL spectrum of the parent clusters.

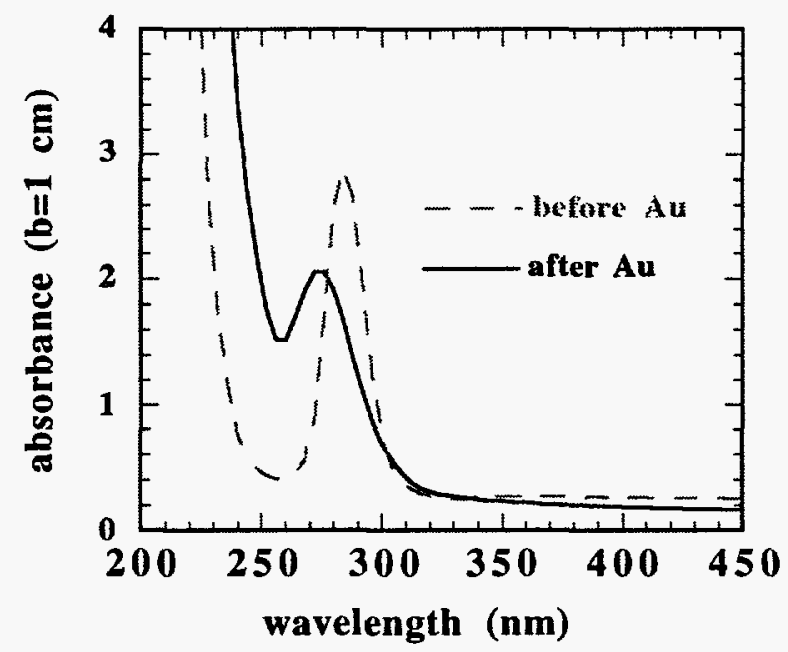

Figure 12. Absorption spectra of $\mathrm{PbS}$ clusters before and after coating with $\mathrm{Au}$.

One may wonder about the details of the surface modification which occurs upon coating with gold. A distinct curved interface cannot be detected in high resolution TEM photographs of these coated nanoclusters where the gold coating 
is only one monolayer thick. In fact, the TEM of Figure 13 is indistinguishable from the parent $\mathrm{PbS}$ clusters (Figure 6). Perhaps with larger clusters and a thicker coating more can be said about the interaction between the two materials.

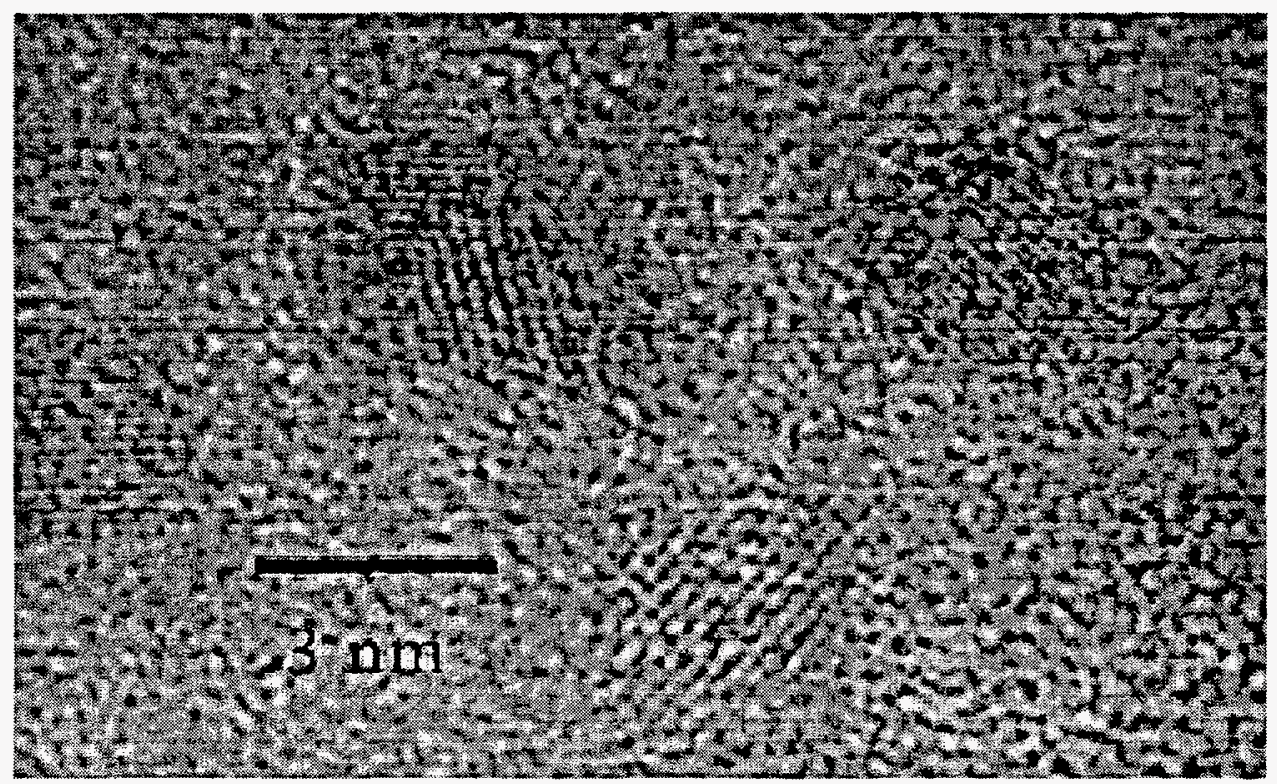

Figure 13. High resolution TEM of gold coated $\mathrm{PbS}$ clusters as described in the text.

\section{F. Conclusions}

In conclusion, we have shown that high quality, size-selected, single crystal $\mathrm{PbS}$ nanoclusters can be successfully grown in inverse micellar solutions. Optical absorption and PL spectra show detailed excitation features similar to diatomic $\mathrm{PbS}$ molecules. The absorption peak gives rise to a non-radiative relaxation which is interpreted to be a dissociative excitation of a surface $\mathrm{PbS}$ molecule. The PL excitation band is interpreted as an excitation of $\mathrm{PbS}$ molecular levels in the interior of the cluster. Results on $\mathrm{Au}$ capping of the $\mathrm{PbS}$ nanoclusters show that surface related states of one material can be excited through states of a different interior material. This effect has extremely important ramifications for photocatalysts: the functions of energy absorption and energy transfer can be 
separated. Materials for the interior of the nanocluster can be chosen to be efficient absorbers of light energy while materials on the surface can be chosen to be efficient at transferring energy to reactant molecules. 


\section{Iron Sulfide (FeS2) Nanoclusters}

\section{A. Motivation}

Our choice of FeS2 was motivated by both applied and scientific interest. On the applied side $\mathrm{FeS}_{2}$ is the major sulfur bearing mineral in coal and is believed to act as a catalyst in coal liquefaction. We have demonstrated significant increases in this catalytic activity for $\mathrm{FeS}_{2}$ nanoclusters.[3] For this LDRD we were interested in these clusters as solar photoelectrochemical catalysts. This is motivated by the ability to tailor the optical absorption to match the solar spectrum by controlling cluster size.

The electronic structures of $\mathrm{FeS}_{2}$ and other $3 \mathrm{~d}$ transition metal dichalcogenides have been of considerable scientific interest because these materials straddle the localization-itinerant regimes for the $3 \mathrm{~d}$ electrons.[9] How this structure varies with electronic confinement would be interesting, but apparently no earlier work exists on this subject. The formal chemical formula of $\mathrm{FeS}_{2}$ is $\mathrm{Fe}^{2+}\left(\mathrm{S}_{2}\right)^{2-}$, and the material crystallizes in the cubic pyrite $\left(\mathrm{T}_{\mathrm{h}}^{6}-\mathrm{Pa} \overline{3}\right)$ structure with 4 molecules per unit cell. The structure can be viewed as rock salt $(\mathrm{NaCl})$-like with $\mathrm{Fe}^{2+}$ ions occupying the cationic sites and $\mathrm{S}_{2}^{2-}$ ion pairs occupying the anionic sites with their diatomic axes ordered equally along the four $<111>$ directions. In this structure, each $S$ is bonded to three $\mathrm{Fe}$ and one $\mathrm{S}$ ions in a distorted tetrahedral configuration, and each Fe ion is surrounded by six $\mathrm{S}$ ions in a distorted octahedral configuration.

\section{B. Synthesis and Characterization}

Our clusters were grown inside inverse micellar cages in non-aqueous solvents.[6] $\mathrm{FeS}_{2}$ clusters are formed by first dissolving an iron halide salt 
inside the cages and then combining this solution with another inverse micelle solution containing a sulfiding agent (e.g., metal sulfide or $\mathrm{H}_{2} \mathrm{~S}$ ). All preparations were done in a Vacuum Atmospheres dry box with catalytic oxygen and water removal to prevent degradation of $\mathrm{Fe}$ salt precursor. Both oxygen and water levels were monitored and kept below $1 \mathrm{ppm}$ during the reaction. The Fe:S ratio was chosen to be $1: 2$ or less. Beautifully clear suspensions varying in color from nearly colorless for the smallest clusters $(<2 \mathrm{~nm}$ in diameter) to deep green or blue/green for the larger clusters $(6 \mathrm{~nm})$ are obtained. The cluster size is varied by using different size micellar cages to encapsulate the Fe salt. These clusters suspensions are remarkable for they are homogeneous, stable and transparent dispersions of $\mathrm{FeS}_{2}$ clusters in a non-polar fluid.

The grown clusters were characterized by a variety of techniques including TEM, x-ray and electron diffraction, optical spectroscopy, dynamic light scattering and small angle $x$-ray and neutron scattering. The selected area diffraction (SAD) pattern (Figure 14) matches that of the cubic pyrite structure of bulk FeS2 and our synthesis produces very little size dispersion (Figure 15). 


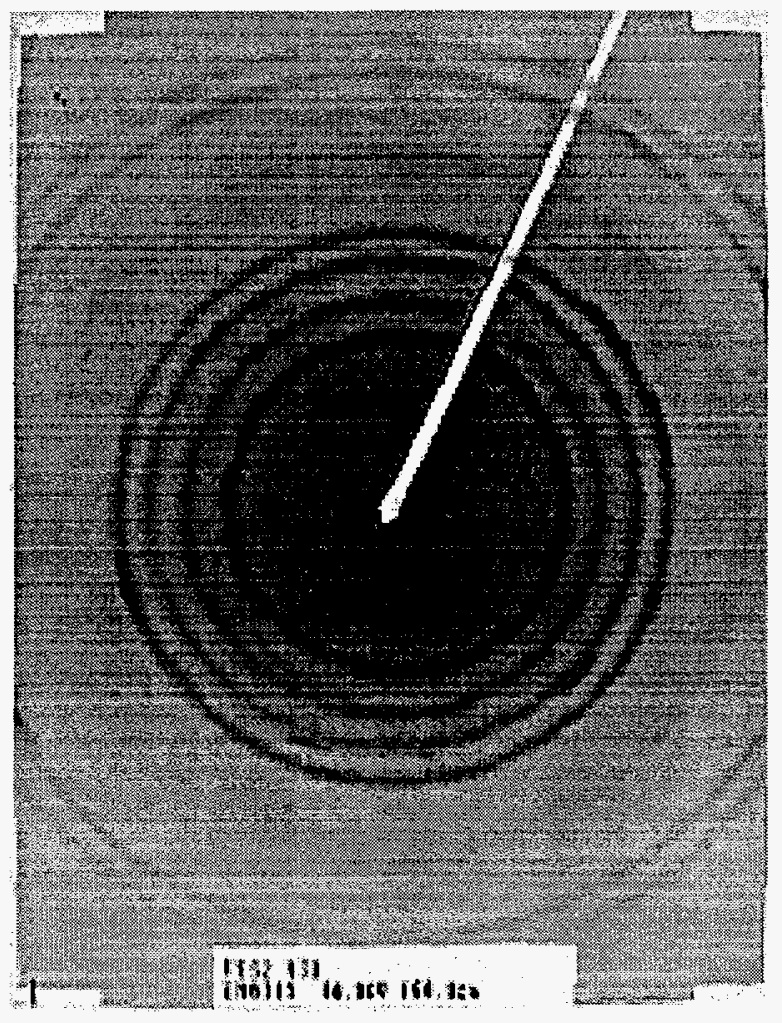

Figure 14. Selected area electron diffraction from a field of $4.5 \mathrm{~nm} \mathrm{FeS} 2$ cluster gives a broad ring pattern which matches cubic pyrite.

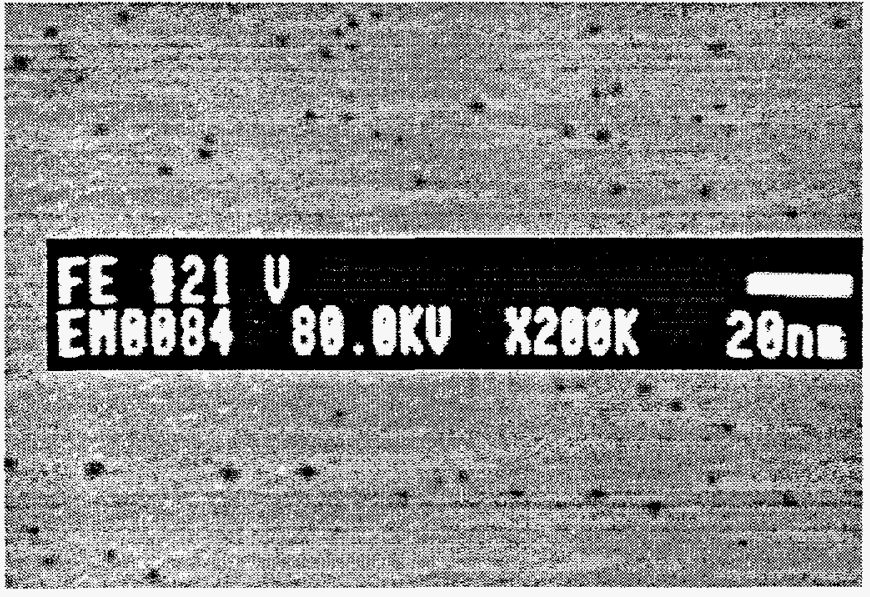

Figure 15. TEM of $2.0 \mathrm{~nm} \mathrm{FeS} 2$ nanoclusters whose absorbance spectrum is shown in Figure 16. 


\section{Optical Properties: Strong Quantum Confinement Effects}

Figure 16 shows the optical absorption spectra of $4.5,3.5 \mathrm{~nm}$ and $2.0 \mathrm{~nm}$ diameter $\mathrm{FeS}_{2}$ clusters dispersed in toluene at concentrations of $10^{-3}$ molar and compares the results with those of Sato[10] on bulk crystalline film. Our spectra, which were measured at $290 \mathrm{~K}$, exhibit well-defined features which we now wish to discuss. A TEM of the $2.0 \mathrm{~nm}$ clusters and an selected area diffraction (SAD) pattern of the largest clusters are shown in Figures 14 and 15 as already noted.

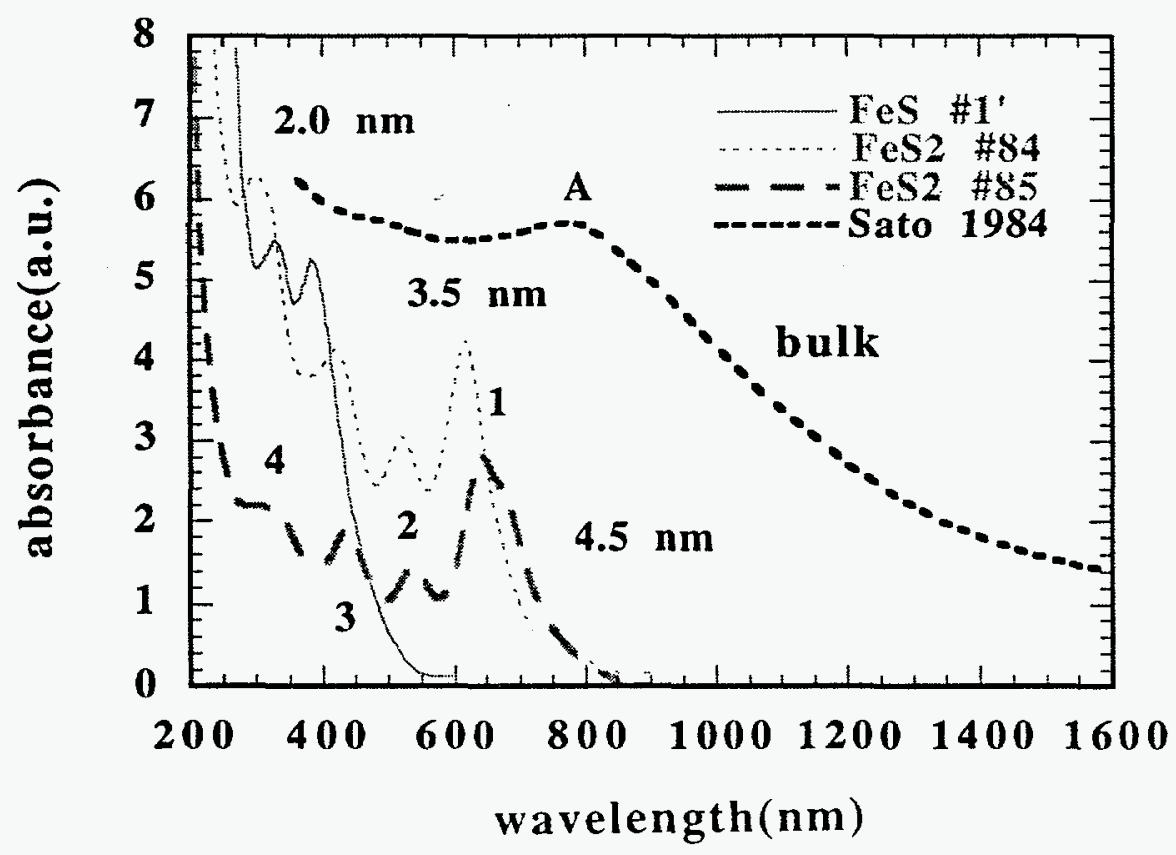

Figure 16. Absorbance spectra (arbitrary units) of $\mathrm{FeS}_{2}$ nanoclusters of the indicated sizes are compared to that of a bulk sample (ref 10).

First, however, it is helpful to review briefly the electronic structure and absorption spectrum of bulk FeS2. Figure 17 a shows the one-electron energy 
level diagram for FeS2 constructed from the correlation of the electronic levels of the outer electrons of the $\mathrm{Fe}^{2+}$ and $\left(\mathrm{S}_{2}\right)^{2-}$ ions.[11] The cubic component of the crystal field splits the Fe $3 \mathrm{~d}$ states into orbitals of $2 \mathrm{~g}$ and eg symmetry. The $\mathrm{t} 2 \mathrm{~g}$ orbitals form a narrow, completely filled, highly localized non-bonding band. One of the $\mathrm{S} \mathrm{sp}^{3}$ orbitals is split into occupied, bonding $\sigma$ orbitals and unoccupied, antibonding $\sigma^{*}$ orbitals. The remaining three $\mathrm{S} \mathrm{sp} 3$ orbitals hybridize with the $\mathrm{Fe}$ e and $4 \mathrm{~s}$ and $4 \mathrm{p}$ orbitals further stabilizing and broadening the $\sigma$ and $\sigma^{*}$ bands. In this picture there are no partially-filled sub-bands, and the bandgap is between the top of the filled $2 \mathrm{~g}$ and the bottom of the empty $\sigma^{*}$ band.

Band structure calculations[9,12] confirm the above picture, and, in agreement with experiment, find the bandgap to be indirect and $\sim 0.9 \mathrm{eV}$ wide. Although there are some quantitative differences, the different calculations agree on the main features. The calculated densities of states (DOS) reveal considerable structure. Figure $17 \mathrm{~b}$ shows the portion of the DOS relevant to the present work. Particularly noteworthy is the structure in the conduction $\left(\sigma^{*}\right)$ band, and more specifically the sharp peak at $3.4 \mathrm{eV}$ above the top of the valence band. 


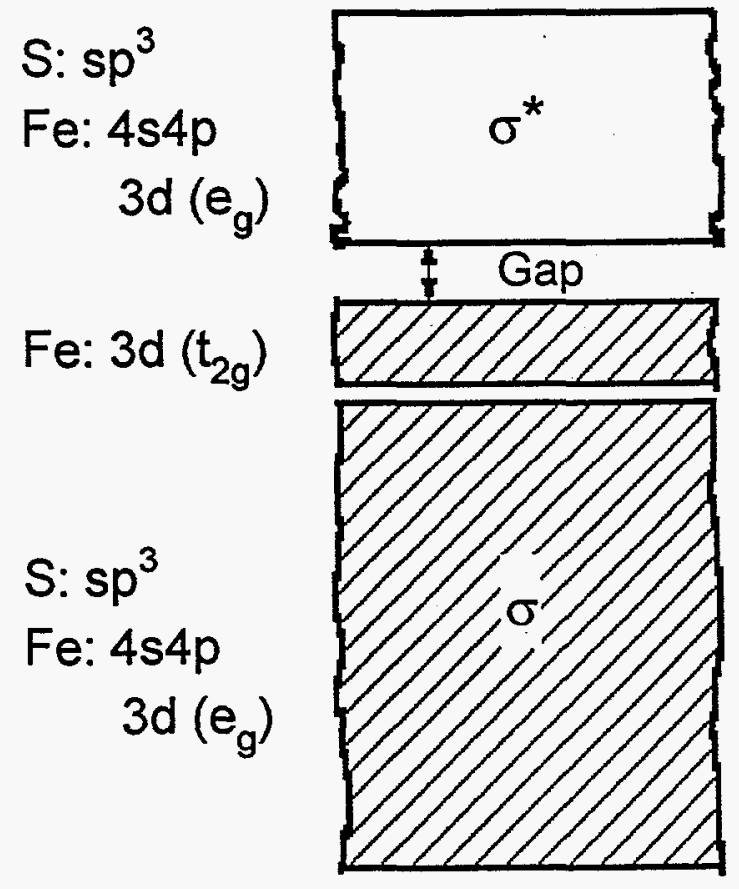

(a)

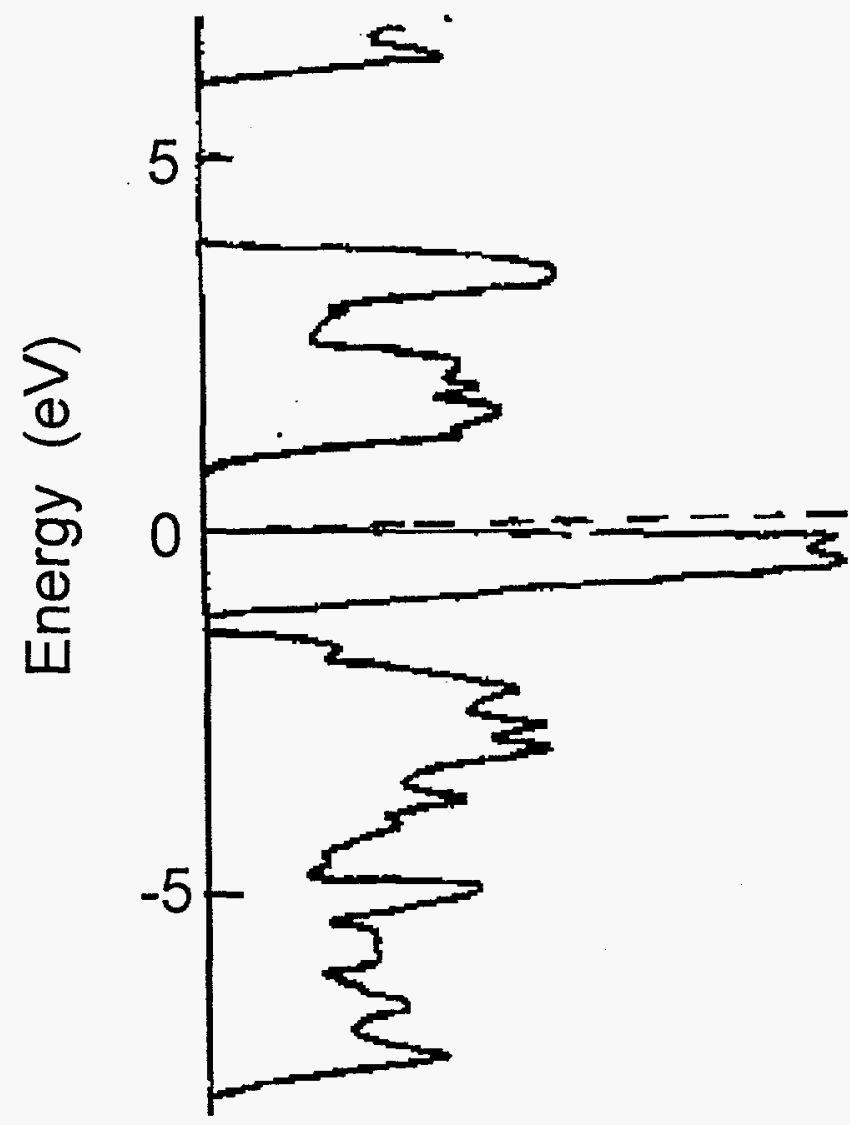

(b)

Figure 17. One electron energy levels for $\mathrm{FeS}_{2}(\mathrm{a})$ and density of states (b) as a function of energy is shown schematically for $\mathrm{FeS}_{2}$ based upon theoretical calculations by Folkerts et al (ref 9). 
The optical properties of bulk FeS2 have been reported by several groups from measurements on natural pyrite crystals, synthetic crystals and crystalline films. Most samples show considerable featureless absorption well below the absorption edge, characteristic of free carrier absorption due to impurities or crystalline defects. These defects and related lack of stoichiometry appear to plague essentially all bulk samples. Most of the reported absorption measurements have been restricted to the region near the absorption edge; however, Sato[10] reported direct absorption measurements that extend to considerably shorter wavelengths $(\lambda)$ on a CVD-grown film as well as an absorption spectrum deduced from reflectivity measurements on a single crystal grown by chemical vapor transport. His results on the film were shown in Figure 16. The extended long $\lambda$ tail is associated with the indirect gap and free carrier absorption. The spectra show two broad bands labeled A (shown in Figure 16) and B (not shown). No explanation is given for the substantial differences in peak locations for the two samples. Band $A$ is assigned to transitions across the gap. Its peak is located at $1.6 \mathrm{eV}$ in the film and at $2.3 \mathrm{eV}$ in the calculated spectrum. Note that the DOS (Figure 17b) has a peak at $1.6 \mathrm{eV}$ above the valence band edge. Band $B$ is believed to be due to transitions from the t2g valence band to the upper parts of the $\sigma^{*}$ (hybridized eg-sp3) band in which p-like character dominates; however, one cannot completely rule out the possibility that it may be due to interband transitions from the deeper $\sigma$ valence band to the lower portion of the $\sigma^{*}$ band. The plateau in band B occurs at $\sim 3.8$ $\mathrm{eV}(325 \mathrm{~nm})$ in the calculated spectrum and we estimate it to be at $\sim 3.5 \mathrm{eV}(355$ $\mathrm{nm}$ ) for the spectrum of the film. Again note that the DOS exhibits a sharp peak at $3.4 \mathrm{eV}$ above the valence band edge. Absorption bands $\mathrm{A}$ and $\mathrm{B}$ are also mimicked in the calculated joint density of states (JDOS) deduced from bandstructure calculations. Folkerts et al [9] find peaks A and B in the JDOS 
spectrum at $\sim 2.5$ and $4.0 \mathrm{eV}$, respectively, whereas the spectrum calculated by Sato[10] on the basis of Bullett's[12] bandstructure results exhibits peaks and plateaus at 1.4 (peak A), 2.2, 2.6 and 2.9 (peak B) eV.

Returning to Figure 16, note that the absorption spectra of the clusters are rich in spectral peaks heretofore not observed in bulk samples. Multiple maxima in the absorption spectra of clusters can be due to either specific electronic transitions in monodisperse clusters or to the presence of structured size distributions (e.g., magic numbers) of clusters in a given sample. In the present case, however, comparison of spectra on several samples shows no evidence for structured size distributions in our samples, and thus the features in the spectrum are intrinsic properties of monodisperse clusters and attest to the high crystalline quality and stoichiometry of our clusters.

Comparison of the spectra in Figure 16 reveals a large blue shift of the absorption edge of the clusters compared to the bulk as well as with decreasing cluster size. The spectrum of the $3.5 \mathrm{~nm}$ clusters has four well-defined peaks labeled 1-4, from lowest to highest energies. The same peaks were observed in many other cluster samples in the 3-5 nm size range. Peak 4 (highest energy) in Figure 16 exhibits some asymmetry, and the spectra on other samples showed that it consists of two closely spaced peaks. It is almost certain that peak 1 corresponds to the peak in band $\mathrm{A}$ of bulk $\mathrm{FeS}_{2}$ and is associated with transitions across the bandgap, but is shifted to the blue by $0.4 \mathrm{eV}$ due to quantum confinement. We conjecture that peak 4 at $4.2 \mathrm{eV}(295 \mathrm{~nm})$ corresponds to the plateau in band B of the bulk, but blue shifted by $\sim 0.5 \mathrm{eV}$. As already noted, there are two possibilities as to the origin of band B: (1) transitions from the $2 \mathrm{~g}$ valence band to the top of the $\sigma^{*}$ band (Figure 17), or (2) interband transitions from the deeper-lying $\sigma$ valence band to the $\sigma^{*}$ band. Although we cannot 
unequivocally distinguish between the two choices at present, the observed intermediate peaks in the cluster spectra (peaks 2 and 3), favor the first choice. In Figure 17 we suggest possible transitions for the first choice which could be responsible for peaks 1-4. The energies of peaks $1-4$ of the $3.5 \mathrm{~nm}$ clusters are 2.0, 2.4, 2.97 and $4.2 \mathrm{eV}(620,517,418$ and $295 \mathrm{~nm})$, respectively, are compatible with the structure in the DOS and JDOS spectra of the $\sigma^{*}$ band (as discussed above) when the $\sim 0.5 \mathrm{eV}$ blue shift due to electron confinement is taken into account. As for peaks 2 and 3, their energies are not compatible with transitions from the $\sigma$ valence band to $\sigma^{*}$, and we suggest they are due to transitions from $\mathrm{t} 2 \mathrm{~g}$ to $\sigma^{*}$.

Decreasing the cluster size from 3.5 to $2.0 \mathrm{~nm}$ causes large blue shifts of the absorption edge as well as of the spectral peaks. Peaks 1 and 2 are still seen in the spectrum of the $2.0 \mathrm{~nm}$ clusters (Figure 16), but peaks 3 and 4 occur at shorter wavelengths than the $\sim 200 \mathrm{~nm}$ limit of the spectrometer used. Clearly also seen in Figure 16 is the significant increase in the absolute absorbance in going from 3.5 to $2.0 \mathrm{~nm}$ clusters, a characteristic feature of small clusters.

The bandgap of bulk $\mathrm{FeS}_{2}$ is known to be indirect with energy $\mathrm{E}_{\mathrm{g}}=0.80$ $\mathrm{eV}$ at room temperature.[13] For an indirect transition the absorption data in the region of the band edge can be described by[13]

$$
\alpha h v=C(h v-E g)^{2}
$$

where $\alpha$ is the absorption coefficient, $h v$ is the photon energy, $\mathrm{C}$ is a constant and $\mathrm{E}_{\mathrm{g}}$ is the bandgap. Absorption data on bulk $\mathrm{FeS}_{2}$ obey this relationship and Figure 18 shows that it also holds for the clusters. In Figure $18(\alpha h v)^{1 / 2}$ vs. hv is plotted for three cluster sizes. The intercepts of the linear responses with the $\mathrm{x}$-axis are the indirect bandgaps for the different sizes. The inset in Figure 18 
shows the shift $(\Delta)$ of $\mathrm{Eg}$ with cluster size. The magnitude of this shift increases with decreasing diameter. For $\mathrm{d}=2.0 \mathrm{~nm}, \Delta=1.4 \mathrm{eV}$, a large effect.

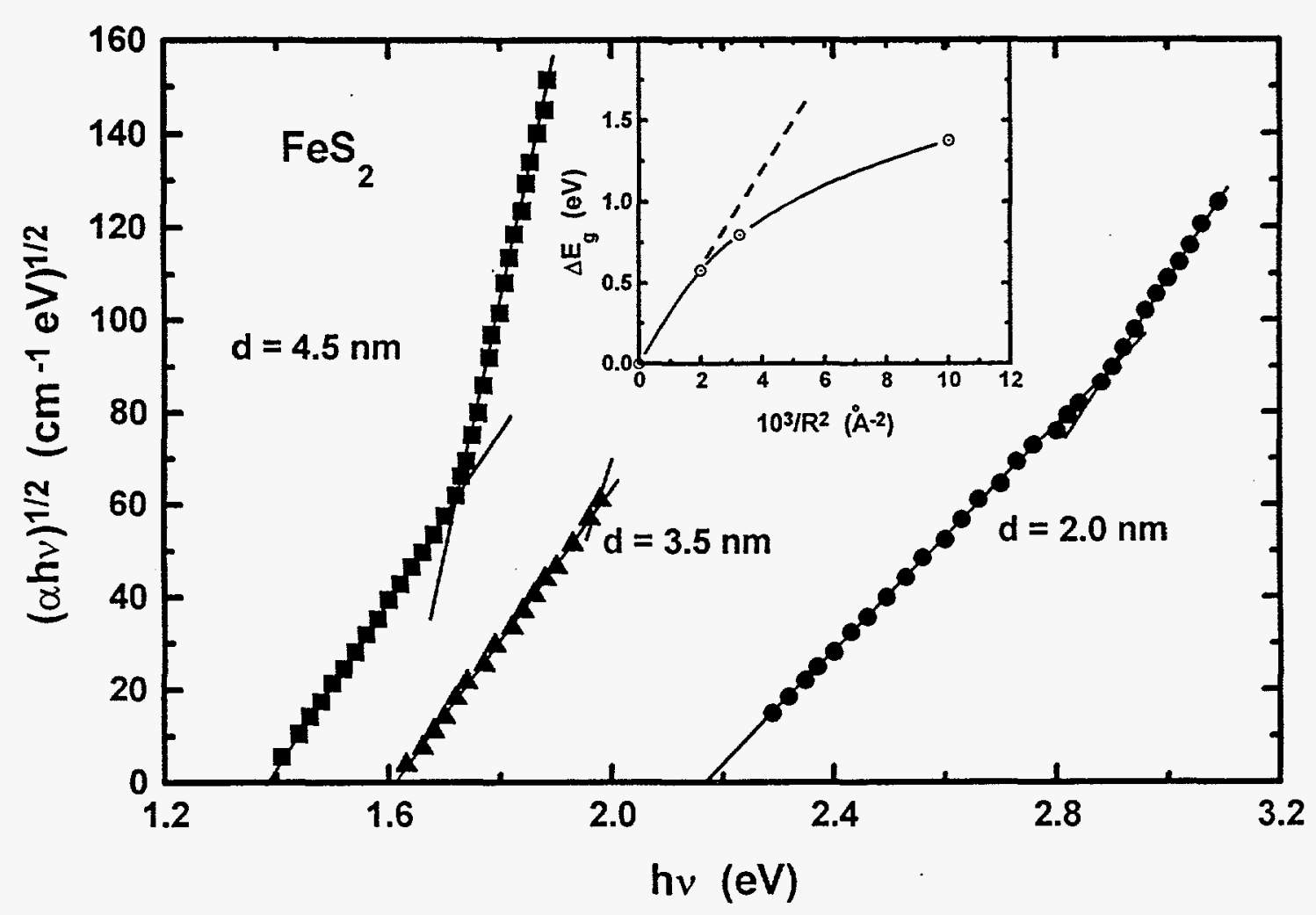

Figure 18. The square root of the photon energy hv is plotted versus hv to obtained the band-edge energy for the three cluster sizes whose spectral data was shown in Figure 16.

In the size regime where quantum confinement effects become prominent effective mass theory predicts that the shift, $\Delta$, of the bandgap with cluster size is to a reasonable approximation proportional to $1 / 2 \mu \mathrm{R}^{2}$, where $\mu$ is the reduced excitonic mass and $\mathrm{R}$ is the (spherical) cluster radius. Thus a plot of $\Delta$ vs. $1 / \mathrm{R}^{2}$ should yield a straight line whose slope is proportional to $1 / 2 \mu$. Such a plot, shown in the inset to Figure 18, shows large deviations from this prediction at 
small cluster sizes, a feature that is commonly observed in semiconductor clusters.

From an analysis of excitonic features on bulk FeS2 crystals, Kou and Seehra[13] found $\mu=0.45 \mathrm{me}_{\mathrm{e}}$, where $\mathrm{m}_{\mathrm{e}}$ is the electronic mass. Using this value of $\mu$ and a value $\varepsilon=10.9$ for the dielectric constant[13] of FeS2, we estimate the excitonic Bohr radius of $\mathrm{FeS}_{2}$ to be $\mathrm{r}_{\mathrm{B}} \approx 13 \AA$. Thus, our $2.0 \mathrm{~nm}$ diameter clusters are a little smaller than the size of the exciton in the bulk, which implies entry into the strong electron confinement regime. In fact, the large increase in $\Delta$, and the correspondingly large shifts of peaks 1 and 2 in the $2.0 \mathrm{~nm}$ cluster spectrum (Figure 16) compared to that of the $3.5 \mathrm{~nm}$ cluster spectrum are consistent with being in this regime. At smaller cluster sizes we expect the optical spectrum to lose its bulk (band)-like features and develop a ladder of molecule-like discrete states. As will be discussed in Sec. V, we have observed such a transition from solid-like to molecule-like behavior in small $\mathrm{MoS}_{2}$ clusters.[14] We shall seek to observe it in future work on FeS2.

\section{Surface Modification}

Iron Sulfide, even in bulk form, is known to be somewhat sensitive to oxidation and often transforms into more thermodynamically stable iron oxides in the presence of molecular oxygen. We find that nanosize $\mathrm{FeS}_{2}$ is even more sensitive to oxygen. To prevent oxidation we initiated a series of experiments in which $\mathrm{FeS}_{2}$ nanoclusters were coated with a monolayer of air-insensitive metals such as gold or palladium. The optical properties and structure of the resulting composites were then studied. The changes in the optical features are shown in Figure 19. The presence of the gold on the surface causes a blue shift of the 
absorption spectral peaks as well as an increase in the absorbance throughout the visible and into the near infrared.

The most important benefit of this coating process in the significant reduction of the rate of oxidation of the $\mathrm{FeS}_{2}$ surface. Figure 20 shows this benefit for the case of palladium coated $\mathrm{FeS}_{2}$ nanoclusters exposed to oxygen for a 24 hour period. In uncoated $\mathrm{FeS}_{2}$ such exposure always results in complete oxidation to iron oxide and loss of all the spectral features shown. However, no optical changes are observed in the coated nanoclusters. This observation indicates that this inexpensive, nontoxic material may have future solar photochemical applications, particularly in detoxification of organic wastes in water.

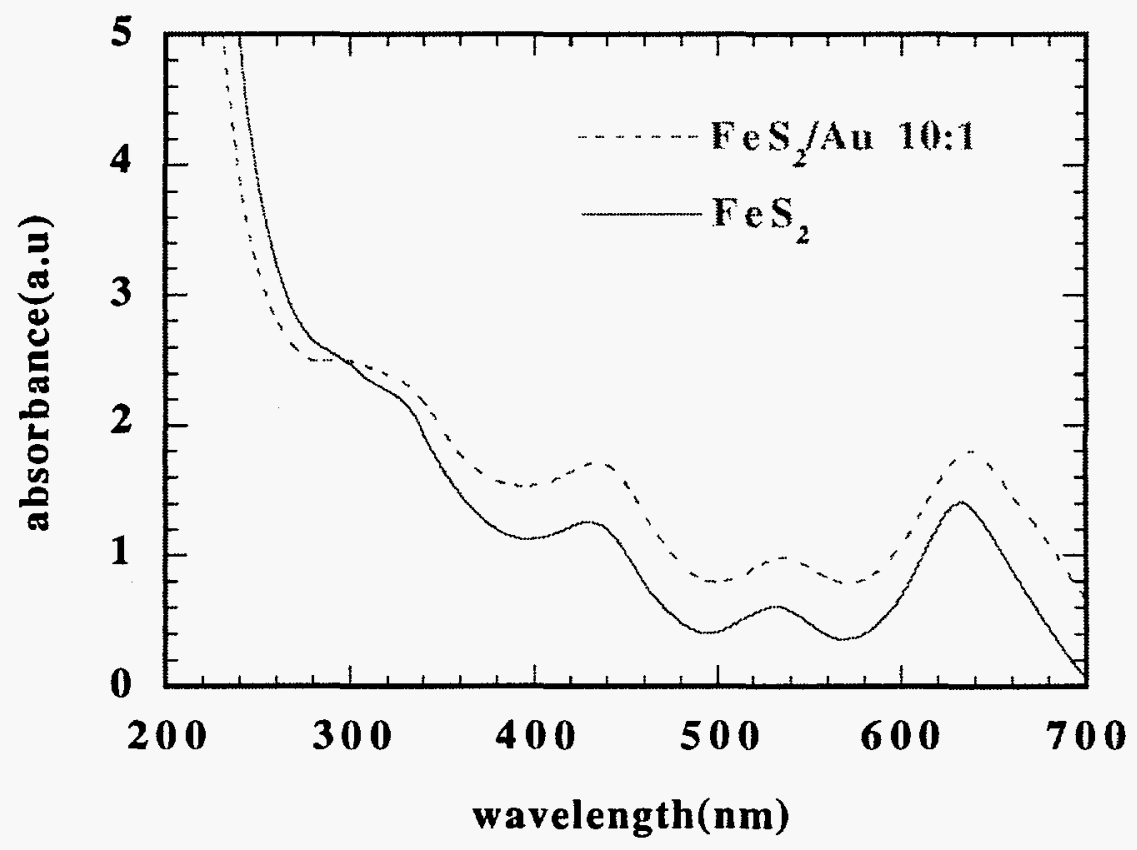

Figure 19. Effect of coating $4.5 \mathrm{~nm} \mathrm{FeS} 2$ nanoclusters with a layer of gold to prevent oxidation of the surface. A blue shift of $\sim 10 \mathrm{~nm}$ is observed in all the semiconductor spectral features. An increase in the absorbance throughout the visible is also observed. 


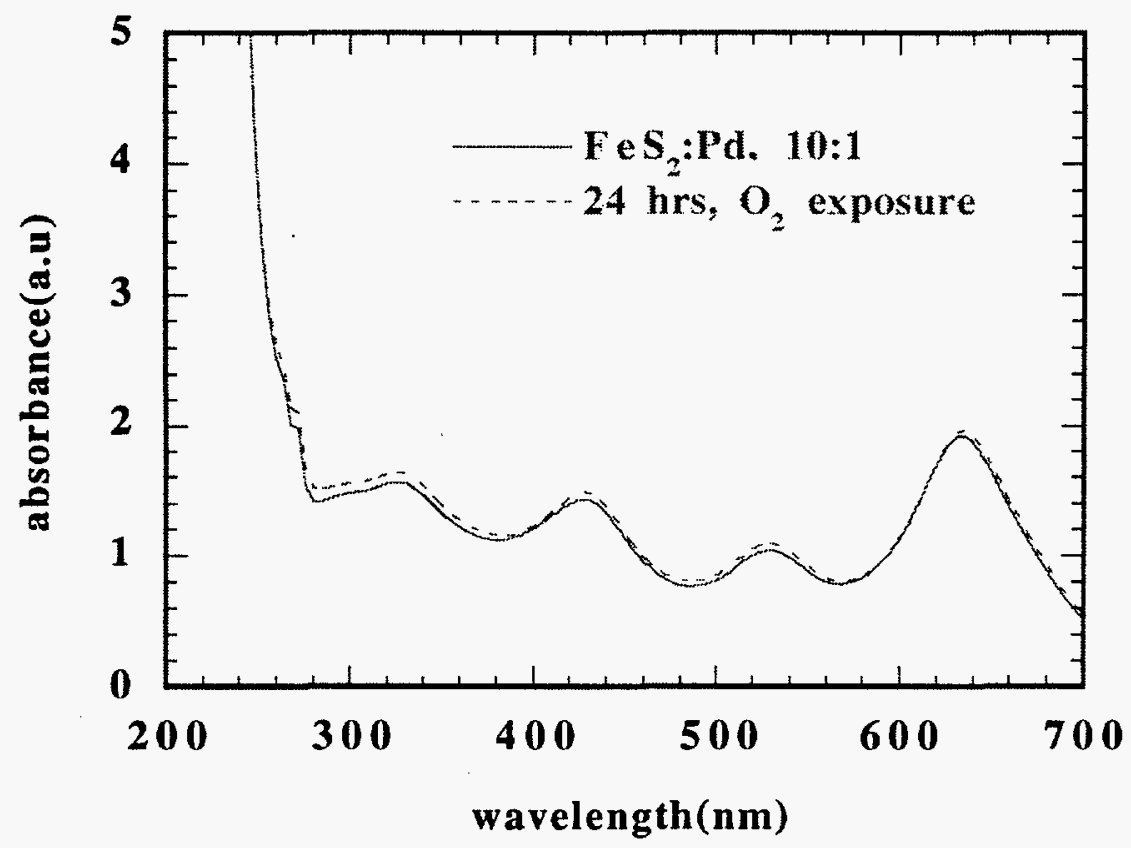

Figure 20. Effect of oxygen exposure on the absorption spectrum of palladiumcoated $\sim 4.5 \mathrm{~nm} \mathrm{FeS} 2$ nanoclusters. Even after $24 \mathrm{hrs}$ of oxygen exposure all spectral features are completely retained without loss of intensity.

\section{E. Conclusions}

This work represents the first successful synthesis of size-selected nano-size clusters of iron sulfide ( $\mathrm{FeS}_{2}$ ) and an understanding of their interesting optical properties. Highlights of the work include the observation of: 1) remarkable heretofore unobserved structure in the absorption spectrum reflecting the underlying physics as well as the high quality and size monodispersity of the clusters; 2) large blue shifts in the spectral features upon crossing from the weak to strong confinement regimes with decreasing cluster size affording great tailorability of the bandgap; and 3) the bandgap remains indirect down to the smallest cluster size studied $(2 \mathrm{~nm})$. We have also demonstrated the ability to 
modify the surface of these nanoclusters by coating them with metals. The metal coating was shown to be effective against oxidation of the clusters -- a feature that is crucially important for the potential application of $\mathrm{FeS}_{2}$ in photocatalysis. 


\section{Molybdenum Sulfide (MoS2) And Isomorphous Layered Semiconductor Nanoclusters}

\section{A. Motivation}

We investigated these novel nanosize materials because they have great promise as photocatalysts. A key issue in their use as photocatalysts or photooxidants for destruction of organic wastes (i.e. solar detoxification) is the ability to adjust their optical and electronic properties to allow the desired absorbance of light and its conversion into hole-electron pairs followed by efficient electron transfer to a molecule in solution (reduction) and/or hole oxidation of another species also in solution. For efficient electron and/or hole transfer, the valence and conduction band levels must match those of the target molecules in solution. This is a formidable task if one has only bulk materials to work with. For example, $\mathrm{TiO}_{2}$ has the ability to oxidize and reduce a wide range of molecules because of its wide bandgap, but its ability to absorb sunlight is also very small because of this wide bandgap. An additional problem noted earlier is that, for typical powders of $\mathrm{TiO}_{2}$, bulk recombination of the hole-electron pair and light emission is vastly favored over the transport of the hole and the electron to the surface where they can be transferred to a solution species. Thus, despite extensive work at Sandia and elsewhere with macroscopic powders of materials such as $\mathrm{TiO}_{2}$ and $\mathrm{CdS}$, efficiencies for either fuel production or solar photooxidation are extremely dismal.

In bulk form $\mathrm{MoS}_{2}$ and its isomorphs (MoSe2, MoTe2, WS $2, \mathrm{WSe}_{2}$ and WTe2) absorb throughout the visible range (i.e. they're black), but their narrow IR bandgaps prevent oxidation and reduction of most materials from occurring. Thus, the ability to shift the valence and conduction band edges and the band-gap through cluster size control and the concomitant quantum confinement effect 
would be a boon to their application as photocatalysts. Our first task was the first synthesis of these materials in nanosize form and the demonstration that the band edge (and the electronic energy levels of the valence and conduction band) could be adjusted by variation of cluster size. Some of our results are presented in Figure 21 where the structured spectra from chromatographically purified clusters are shown. These spectra reflect the very different electronic properties of these materials and demonstrated the great tailorability of the optical properties with changing cluster size.

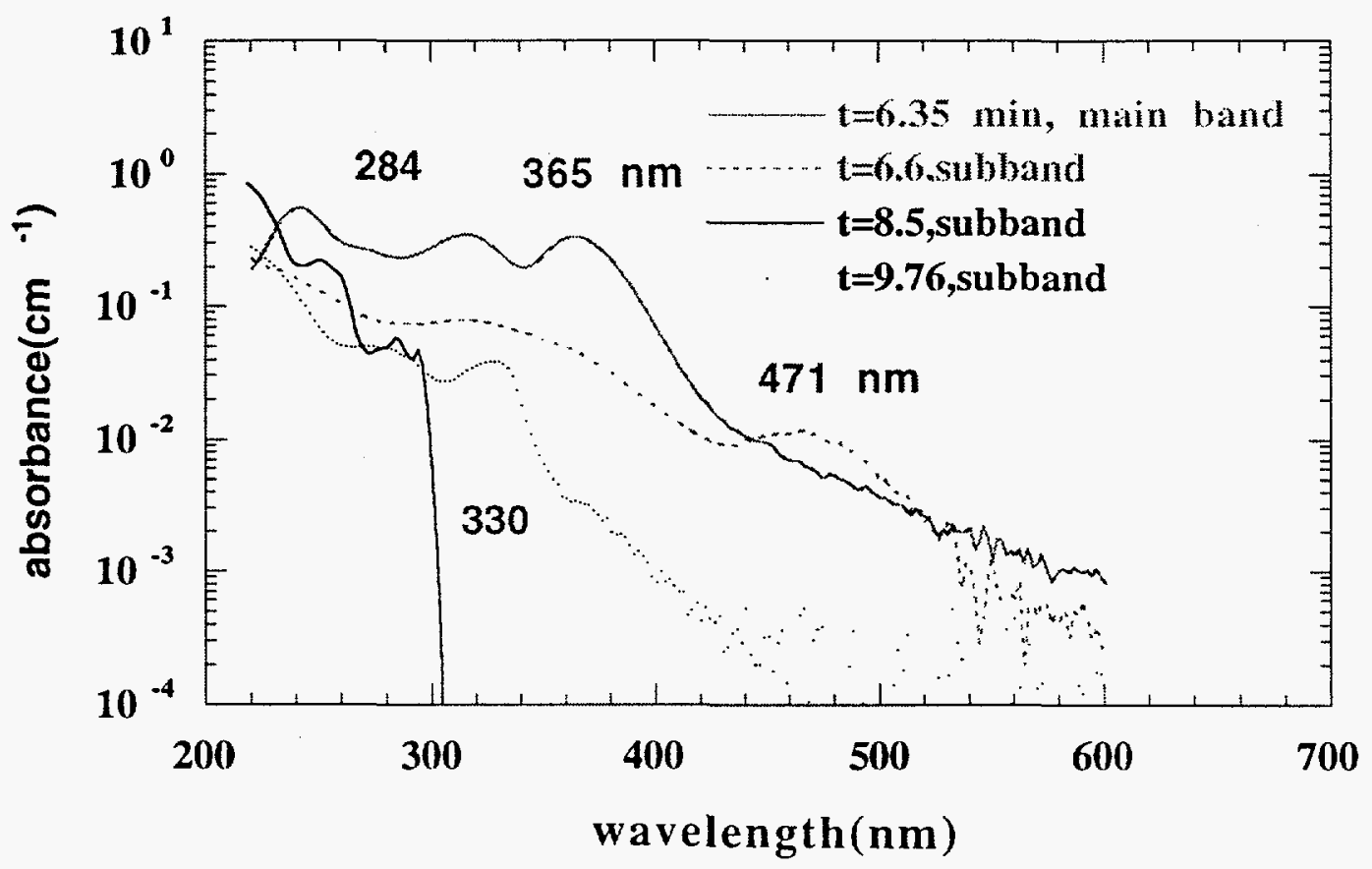

Figure 21. The bandgap of $\mathrm{MoS}_{2}$ shifts dramatically with size for $\mathrm{MoS}_{2}$ clusters. The first excitonic peak is shown in each case.

In size exclusion chromatography, the retention time $t$ scales with the log of the cluster diameter which, for the spectra shown in Figure 21, ranges from $4.5 \mathrm{~nm}$ to $<1 \mathrm{~nm}$. Larger clusters are excluded more effectively from the pore structure of the column materials and thus take less time to elute from the 
column. We note that all of the clusters whose spectra are shown in Figure 21 are expected to have very favorable oxidation and reduction potentials under solar irradiation.

A significant and, as yet not understood feature, of $\mathrm{MoS}_{2}$ and its isomorphs is the way the two-dimensional structure of these materials influences the electronic properties. Bulk $\mathrm{MoS}_{2}$ crystallizes in a hexagonal layered structure (P63/mmc-D $\left.{ }^{4} 6 h\right)$ composed of sheets of Mo atoms sandwiched between sheets of $\mathrm{S}$ atoms in a S-Mo-S arrangement. The structure is depicted schematically in Figure 22. The S-Mo-S sandwiches are held together by weak van der Waals forces and are stacked along the $c$-axis with two sandwiches per unit cell ( $c=12.3$ $\AA$ ). Because of this anisotropy a relevant and important question is which dimension controls the quantum confinement effects shown in Figure 21? The transverse cross-section as measured by both size-exclusion chromatography, light scattering and TEM or the thickness of the nanocluster? In fact, the careful reader will note from the above Figure 21 that the second cluster band at 6.6 minutes actually has a lower energy exciton that the one eluting at 6.35 minutes. If one interprets the shorter elution time as representative of larger overall cluster volume, (the characteristic which controls the elution time and makes larger objects elute earlier than smaller ones), this figure suggests that the thickness of the clusters at 6.35 minutes might be less than those at 6.6 minutes, but have a larger cross-section, and thus a larger overall volume. The question is particularly relevant since recent STM measurements by our collaborator David Kelley at Colorado State have indicated some clusters are only one sandwich layer thick but are significantly larger in the orthogonal directions. 

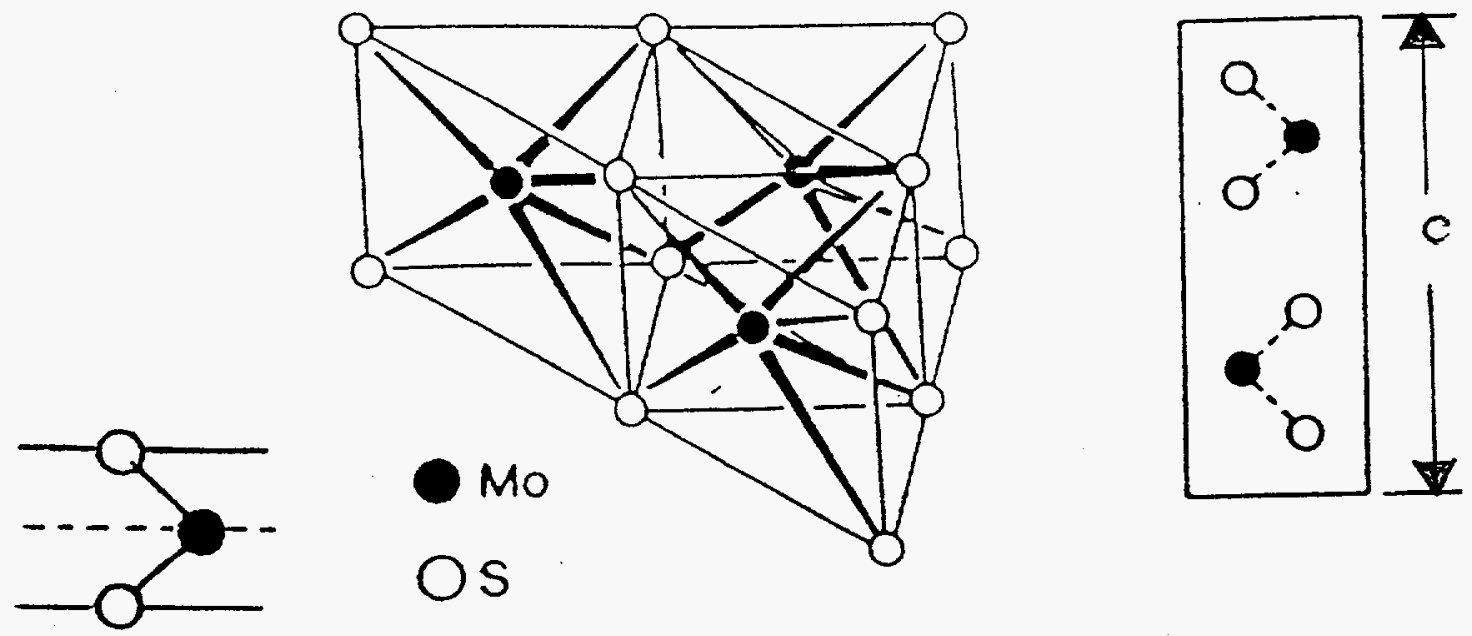

Figure 22. Schematic of the two-dimensional sandwich structure of MoS2.

\section{B. Synthesis and Characterization of $\mathrm{MoS} 2$}

Our clusters were grown inside inverse micellar cages in non-aqueous solvents. Although we focus on $\mathrm{MoS}_{2}$ as a model system, we expect the other isomorphs mentioned above to behave similarly. $\mathrm{MoS}_{2}$ clusters are formed by first dissolving a molybdenum (IV) halide salt inside the cages and then combining this solution with another inverse micelle solution containing a sulfiding agent (e.g., metal sulfide or $\mathrm{H}_{2} \mathrm{~S}$ ). All preparations were done in a Vacuum Atmospheres dry box with catalytic oxygen and water removal to prevent degradation of the Mo (IV) salt precursor. Both oxygen and water levels were monitored and kept below $1 \mathrm{ppm}$ during the reaction. The Mo:S ratio was chosen to be 1:2 or less.[14] Beautifully clear suspensions varying in color from nearly colorless for the smallest clusters $(<2 \mathrm{~nm}$ in diameter) to deep blue for the larger clusters $(\sim 15 \mathrm{~nm})$ are obtained. The cluster size is varied by using different sized micellar cages to encapsulate the Mo salt. These cluster suspensions are remarkable for they are homogeneous, stable and transparent dispersions of $\mathrm{MoS}_{2}$ clusters in a non-polar fluid. When the reaction 
stoichiometry exceeds $2: 1$ in S:Mo then electron diffraction and x-ray diffraction show the expected bulk hexagonal structure as shown in the SAD pattern of Figure 23. However, studies of crystal structure on clusters with sizes less than 3 $\mathrm{nm}$ are very difficult as there are simply not enough atoms in bulk lattice positions to give a significant diffraction signal, nor many reflections. Thus, the structure of the smallest, molecular-like clusters remains unresolved. High resolution TEM fringe imaging reveals that clusters of $\sim 3 \mathrm{~nm}$ size are highly crystalline with little or no crystalline defects. An example of $3.2 \mathrm{~nm}$ clusters is shown in Figure 24.

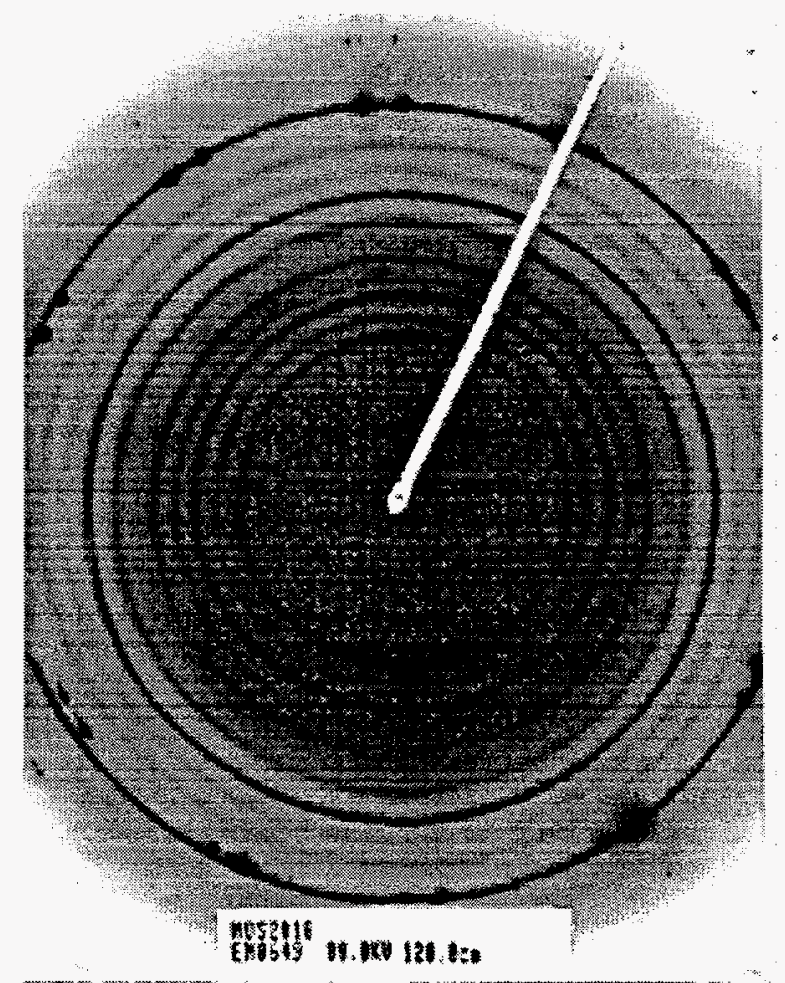

Figure 23. SAD of $4.5 \mathrm{~nm} \mathrm{MoS} 2$ clusters grown in an inverse micelle solution shows the broad diffuse rings and lattice symmetry expected for a hexagonal structure. 


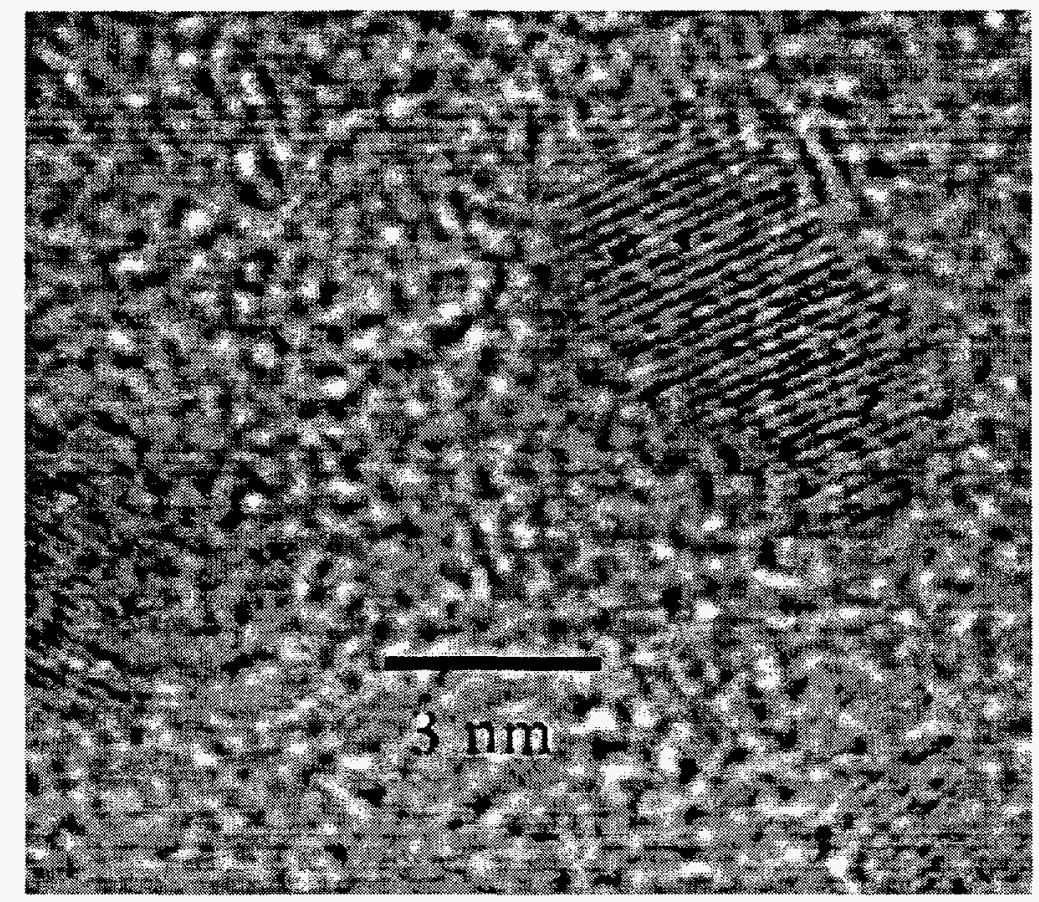

Figure 24. High resolution image of $3.2 \mathrm{~nm} \mathrm{MoS} 2$ clusters shows the excellent crystal order and lack of point and line defects.

\section{Optical Properties and the Crossover from Weak to Strong Quantum}

\section{Confinement}

The optical absorption spectrum of bulk and thin film $\mathrm{MoS}_{2}$ has been studied extensively $[15,16]$ and compared with results of electronic bandstructure calculations [17]. Figure 25 shows room temperature results on two crystalline thin films taken from references 15 and 16. The spectra have well defined spectral features which we shall discuss and use as a reference for comparison with our cluster spectra. The spectra consist of a series of absorption thresholds the first of which corresponds to weak absorption in the near IR at $\sim 1040 \mathrm{~nm}(\sim$ $1.2 \mathrm{eV}$ ) (not shown) associated with an indirect gap between $\Gamma$ and the middle of the Brillouin zone between $\Gamma$ and $K$ (represented by the arrow in the inset in Figure 25). The second threshold occurs at $\sim 700 \mathrm{~nm}$ and is associated with a direct transition at the $\mathrm{K}$ point. The two sharp peaks ( $\mathrm{A}$ and $\mathrm{B}$ ) on the high energy side of this threshold are excitonic transitions ( $\mathrm{K}_{4} \leftrightarrow \mathrm{K}_{5}$ and $\mathrm{K}_{1} \leftrightarrow \mathrm{K}_{5}$, 


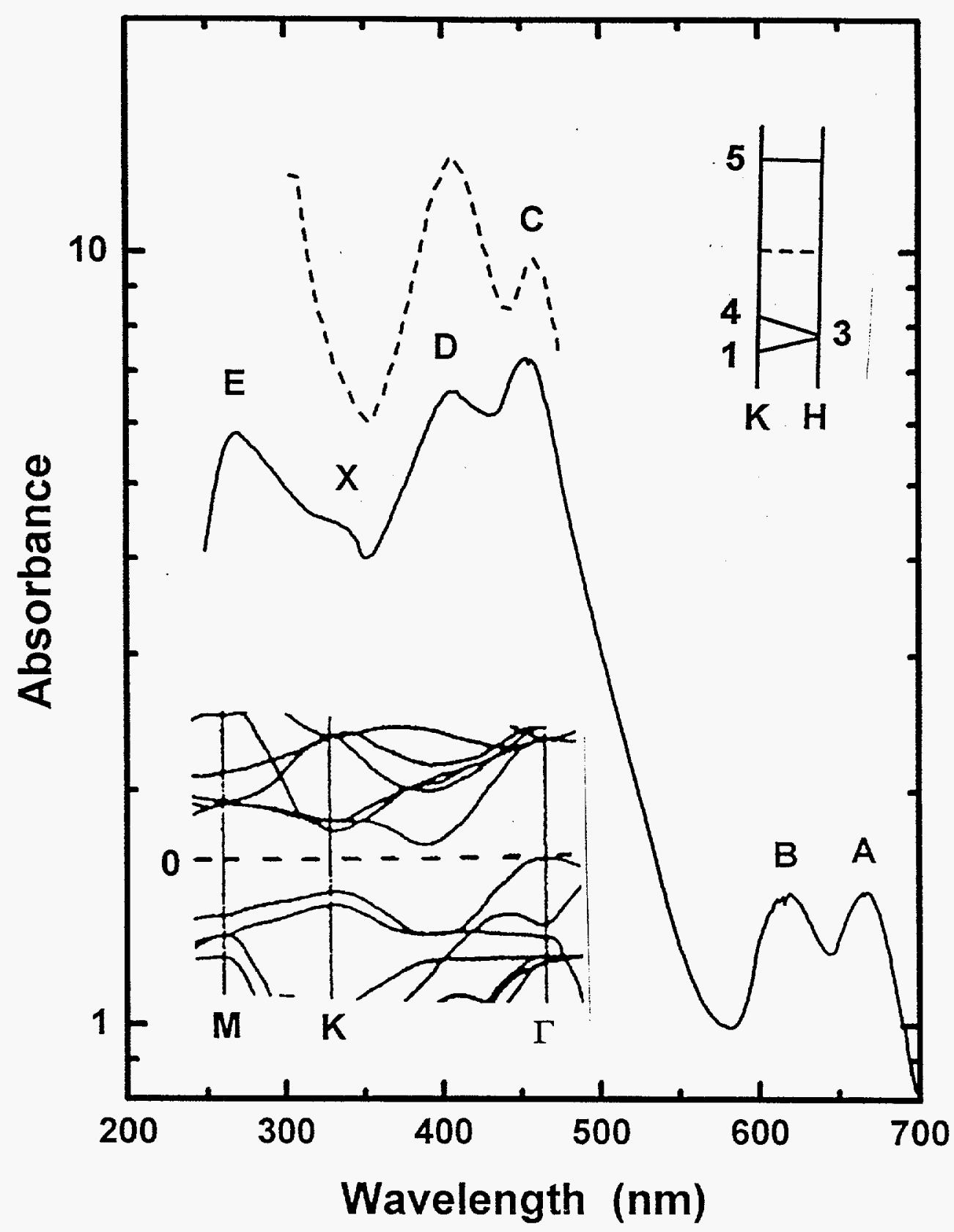

Figure 25. Room temperature optical absorption spectra of two crystalline thin films of $\mathrm{MoS}_{2}$ taken from Refs. 15 and 16. The inset shows a portion of the energy band structure taken from Ref. 17. See text for details. 
respectively) whose energy separation $(\sim 0.20 \mathrm{eV})$ is due to the spin-orbit splitting of the top of the valence band at the K point.[17] A third threshold at $\sim 500 \mathrm{~nm}$ is due to a direct transition (specifics unknown) from deep in the valence band to the conduction band. Excitonic features (C and D) are also associated with these transitions. A fourth threshold at $\sim 350 \mathrm{~nm}$ is also due to transitions from deep in the valence band. Features on the high energy side of this threshold (labeled $X$, our own notation, and E in Figure 25) are seen in thin samples. The relative intensities of peaks $\mathrm{A}$ and $\mathrm{B}, \mathrm{C}$ and $\mathrm{D}$, and $\mathrm{X}$ and $\mathrm{E}$ are known to vary with sample thickness as well as quality $[15,16]$.

Figure 26 shows the room temperature absorption spectra of $4.5 \mathrm{~nm}$ and $2.5 \mathrm{~nm}$ diameter MoS2 clusters dispersed in acetonitrile at a concentration of $\sim 10^{-3}$ molar. These spectra are rich in spectral features which we now wish to discuss. We begin with the spectrum for the $4.5 \mathrm{~nm}$ clusters and have labeled its most prominent features 1 through 5 . Multiple maxima in the absorption spectra of clusters can be due to either specific electronic transitions in monodisperse clusters or to the presence of structured size distribution (e.g., magic numbers) of clusters in a given sample. In the present case, however, comparison of spectra on several samples shows no evidence for structured size distributions, and thus the features in the spectrum are intrinsic properties of monodisperse clusters and attest to the high quality of our clusters as was shown in the lattice fringe images of Figure 24.

Comparison of the spectrum of the $4.5 \mathrm{~nm}$ clusters to that of the bulk crystalline film reveals two main features: (1) a large blue shift for the clusters, and (2) preservation of the excitonic features in the spectrum of the clusters. In fact, a detailed comparison of this spectrum to that of the $50 \mathrm{~nm}$ thick film allows an essentially one-to-one association of the features in both spectra. First, it seems certain that feature $1(\lambda=470 \mathrm{~nm})$ in the cluster spectrum is associated 


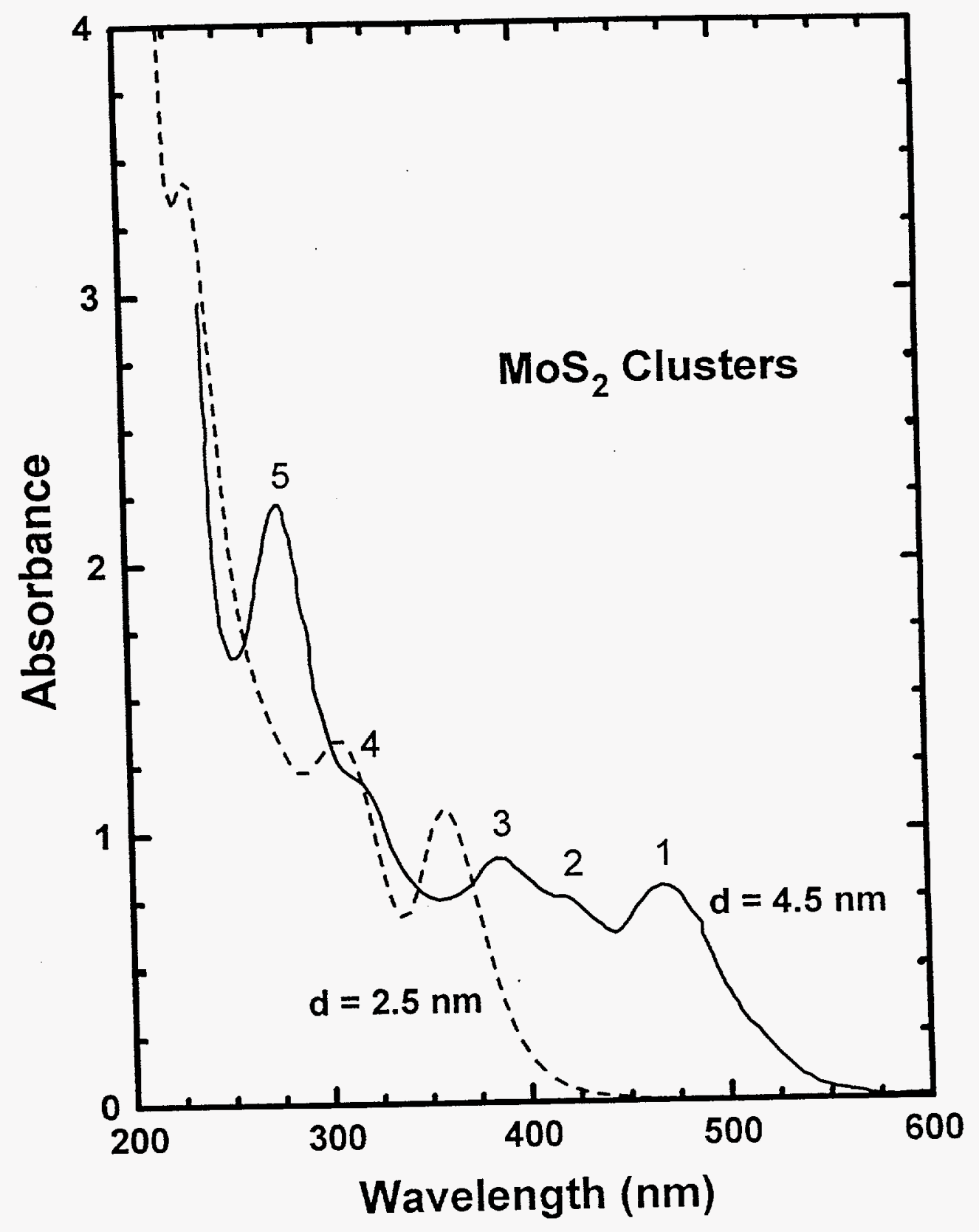

Figure 26. Optical absorption spectra of 4.5 and $2.5 \mathrm{~nm} \mathrm{MoS} 2$ clusters (absorbance in arbitrary units). 
with excitonic peak $A(\lambda=665 \mathrm{~nm})$ of the bulk, corresponding to a blue shift of $0.78 \mathrm{eV}$ due to the quantum size confinement effect of the $4.5 \mathrm{~nm}$ clusters. If we then, as a rough approximation, assume that all of the other peaks of the bulk crystalline spectrum are blue shifted by the same amount, then their energies in $\mathrm{eV}(\mathrm{nm})$ would be: $\mathrm{B}=2.86$ (434), $\mathrm{C}=3.45$ (360); $\mathrm{D}=3.90$ (318), $\mathrm{X}=4.50$ (276), and $E=5.30$ (234). For comparison, features 2, 3, 4, and 5 in the spectrum of the $4.5 \mathrm{~nm}$ clusters have energies of: 2.92 (425), 3.24 (382), 3.92 (317) and 4.51(275),respectively. It is seen that these latter energies are close to the expected shifted positions of peaks $\mathrm{B}, \mathrm{C}, \mathrm{D}$ and $\mathrm{X}$ (the biggest difference being for peak C), thereby providing an argument for the one-to-one association of these features. Our assumption that all the peaks in the single crystal spectrum blue shift equally in energy upon going to the clusters cannot, of course, be strictly correct as different parts of the band structure (or Brillouin Zone) should be affected differently by quantum size confinement due to differences in effective masses. In reality, however, the above analysis shows that this assumption is not far wrong for $\mathrm{MoS}_{2}$ which implies, in the context of simple models, that the effective mass must not vary strongly among the various points of the Brillouin Zone involved in the observed optical transitions. Despite any uncertainties in the above association of spectral features, the important point we wish to make is that the absorption spectrum of the $4.5 \mathrm{~nm}$ clusters is essentially bulk like.

The above conclusion that features 1 to 5 in the spectrum of the $4.5 \mathrm{~nm}$ clusters derive from features in the bulk single-crystal spectrum is consistent with the fact that these clusters are somewhat larger than the size of the exciton (Bohr radius $r_{B}=2.0 \mathrm{~nm}$ ) in bulk $M_{2}$. For these clusters there are apparently sufficiently high densities of states in the conduction and valence bands to make it possible to produce bulk band-like features in the absorption spectrum and 
thereby preserve the quasiparticle character of the excitons. Thus, the spectrum of the $4.5 \mathrm{~nm}$ clusters reflects in a beautiful manner size quantization of excitonic features in the weak-to-moderate confinement regime (cluster radius $R>r_{B}$ ). The above results also strongly indicate that $4.5 \mathrm{~nm} \mathrm{MoS} 2$ clusters retain the bulk hexagonal crystal structure of this material, a feature confirmed by high resolution TEM images.

Turning next to the spectrum of the $2.5 \mathrm{~nm}$ clusters in Figure 26, we note that this spectrum is qualitatively different from that of the $4.5 \mathrm{~nm}$ clusters and of the bulk crystal. Specifically, on going to the $2.5 \mathrm{~nm}$, clusters, the excitonic features in the spectrum give way to a ladder of three well-defined peaks over the spectral range covered. This is the expected behavior in crossing over from the weak (excitonic) confinement regime to the strong (non-interacting carrier) confinement regime where the cluster size (in this case $R=1.25 \mathrm{~nm}$ ) is considerably smaller than the excitonic Bohr radius $\left(r_{B}=2 n m\right)$. For these very small clusters the low densities of states prevents observation of excitonic features, and the allowed energy levels change from continuous bands to a ladder of essentially discrete levels as in molecular systems. We find that the spectrum of $3.0 \mathrm{~nm} \mathrm{MoS} 2$ clusters is very similar to that of the $2.5 \mathrm{~nm}$ clusters reflecting this ladder of discrete levels. This result indicates that the crossover from solidlike to molecule-like behavior occurs at a cluster size between 4.5 and $3.0 \mathrm{~nm}$.

The ladder of peaks in the absorption spectra of the clusters in the smaller than $3 \mathrm{~nm}$ size range then corresponds to transitions to various excited molecularlike states with some vibrational broadening.

A variety of theoretical models have been used to describe size quantization of the energy spectrum of the carriers in semiconductor clusters. These models, which express the size dependence of the energy of the excited states in terms of the bulk energy gap, the kinetic energy, Coulomb interaction and correlation 
energy of the electron-hole (e-h) pairs, have been recently reviewed by Yoffe.[23] They include the initial effective mass (EM) treatment of Efros and Efros[19] and later improvements by Brus,[20] Kayanuma[21] and others, semiempirical tight binding treatments and recent empirical pseudo potential methods [23]. Despite their shortcomings, the EM models contain some of the essential physics and provide a qualitative framework for examining experimental data and their trends. In terms of these models, the ground state energy of a cluster of radius $\mathrm{R}$ is given by [23],

$$
\mathrm{E}(R)=\mathrm{E}_{g}+\frac{h^{2} \pi^{2}}{2 \mu R^{2}}-\frac{1.786 e^{2}}{\varepsilon R}-0.248 \mathrm{E}_{R},
$$

where Eg is the bandgap of the bulk crystal, $\mu$ is either the reduced mass of the exciton (in the weak confinement regime) or the electronic effective mass (in the strong confinement regime), $\mathrm{e}$ is the electronic charge, $\varepsilon$ is the dielectric constant of the cluster, and $E_{R}$ is the Rydbergy energy (equal to the bulk exciton binding energy). The second term on the right-hand side is the kinetic energy term which represents the quantum localization energy, and the third and fourth terms are the Coulomb and correlation energies, respectively, which are usually relatively small [23]. It can be seen that with decreasing $R$ the kinetic energy term becomes increasingly dominant. For our small $\mathrm{MoS}_{2}$ clusters we estimate it to represent over $80 \%$ of the total energy shift. Assuming this term to be the dominant energy over the range of cluster sizes examined, a plot of $E(R)$ vs. $1 / R^{2}$ should yield a straight line with slope $\alpha 1 / 2 \mu$. Such a plot for the sharp absorption threshold at the $\mathrm{K}$ point $(\mathrm{Eg}=1.74 \mathrm{eV}$ in the bulk) for our clusters is shown in Figure 27 and is linear for clusters larger than $3 \mathrm{~nm}$ in diameter. The linear portion yields $\mu=0.16 \mathrm{~m}_{0}$, where $\mathrm{m}_{0}$ is the free-electron mass. This value compares favorably with the bulk value [16] of $0.18 \mathrm{~m}_{0}$ for the A exciton, which emphasizes that the model captures the essential physics. 


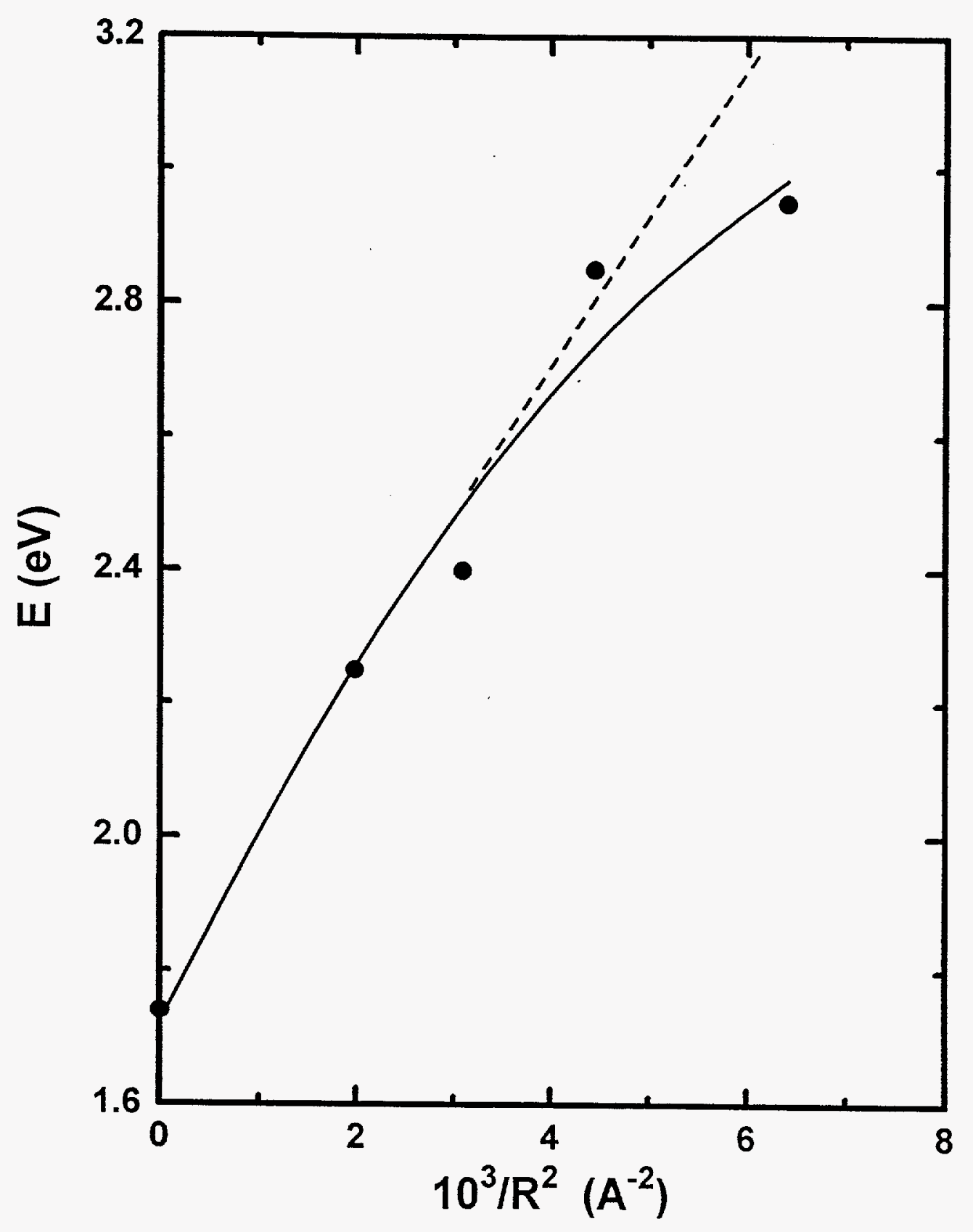

Figure 27. Variation of the absorption threshold energy at the $\mathrm{K}$ point of the Brillouin zone with cluster size for $\mathrm{MoS}_{2}$ clusters. $\mathrm{R}$ is the cluster radius. 
In the strong confinement regime (where the electrons and holes can be regarded as free particles so that their Coulomb and correlation energies can be neglected), the possible quantized e-h pair energies (i.e., the ladder of allowed states) for a particle in a spherical box with infinite barriers can be written as $[19,23]$,

$$
\mathrm{E}_{l, n}=\mathrm{E}_{g}+\frac{h^{2}}{2 m_{e}^{*} R^{2}} \phi_{l, n,}^{2}
$$

where $\mathrm{me}^{*}$ is the effective mass of the electron and $\phi_{l, n}$ is the $n$th root of the spherical Bessel function of $l$ th order with $n$ and $l$ the principal quantum numbers taking the values $n=1,2,3, \ldots$ and $l=0,1,2, \ldots$ For the lowest $1 \mathrm{~s}$ excited state $\phi_{0,1}=\pi=3.14$ and $\phi_{1,1}=4.49$ and $\phi_{2,1}=5.76$. If we take these $\phi_{l, n} s$ to represent the first, second and third peaks in order of decreasing $\lambda$ for the spectrum of the $2.5 \mathrm{~nm}$ clusters in Figure 26, we find that Eq. (2) overestimates the energies for all the peaks of these very small clusters (see, e.g., Figure 27), a situation akin to that for II-VI semiconducting clusters [23]. This discrepancy between experimental and theoretical results for these energies is believed to be due to shortcomings in the model, namely the neglect of the nonparabolicity of the energy vs. wavevector relation and the assumption of infinite barriers at the surface of the cluster. The shape and structure of the clusters (see below) are undoubtedly also important factors as it is almost certain that $\mathrm{MoS}_{2}$ clusters in this size range are not spherical and the lattice distortion must be quite substantial.

Figure 28 shows the room temperature absorption spectrum and the emission spectrum excited at $476 \mathrm{~nm}$ for the $3.0 \mathrm{~nm}$ diameter clusters. In this early study we did not observe any luminescence due to direct e-h recombination near the absorption threshold for any of the clusters. Rather, the spectrum 
consists of a blue-shifted (relative to bulk emission), broad emission peak centered at $537 \mathrm{~nm}$ which is characteristic of surface and/or defect recombination. For these $3.0 \mathrm{~nm}$ clusters many of the atoms lie at or near the surface, the surface states associated with adsorbed species dangling bonds and other defects can be expected to play a major role in determining the fate of the carriers produced by light absorption.

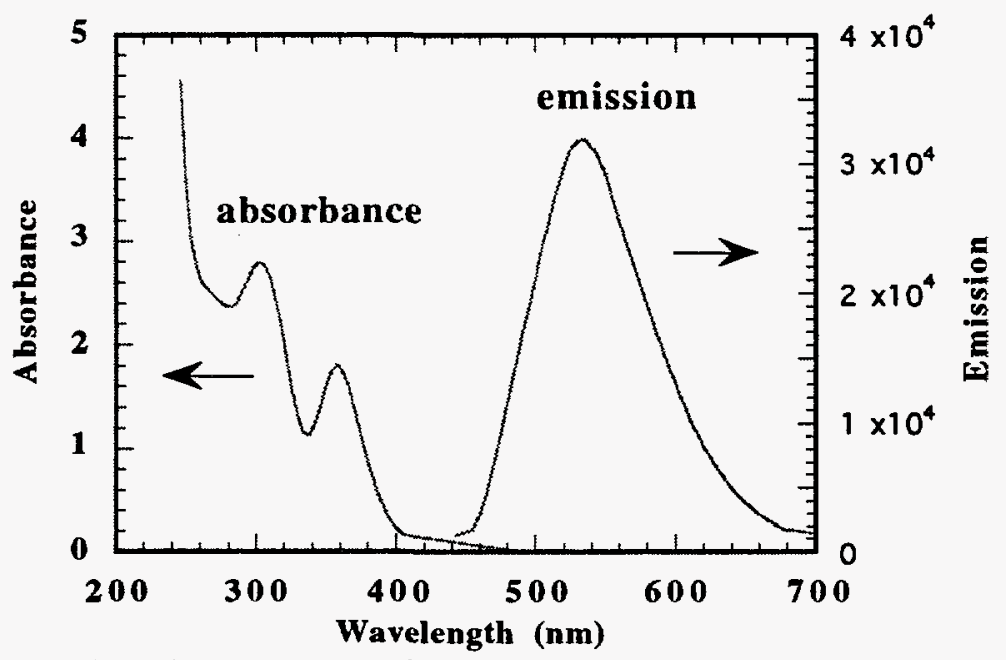

Figure 28. Photoluminescence from $3.0 \mathrm{~nm} \mathrm{MoS} 2$ clusters is broad and relatively weak and is due primarily to recombination by surface states. Little difference in the absorbance or photoluminescence is observed compared to the $2.5 \mathrm{~nm}$ clusters of Figure 26.

Finally, given the layered nature of the $\mathrm{MoS}_{2}$ structure, it is interesting to speculate about the shape of $\mathrm{MoS}_{2}$ clusters in the size range of the present study. In its hexagonal phase, the unit cell of $\mathrm{MoS}_{2}$ contains 2 formula units and has dimensions $a=3.15 \AA$ and $c=12.30 \AA$ yielding a unit cell volume $=317 \AA^{3}$. Assuming spherical clusters and full packing, a $4.5 \mathrm{~nm}$ diameter cluster would contain 150 unit cells, or 300 formula units, and a $2.5 \mathrm{~nm}$ diameter cluster would contain 26 unit cells, or 52 formula units. However, the shape of the unit cell 
precludes full packing, and so the number of molecules in these clusters can be expected to be considerably less. As noted above, the $4.5 \mathrm{~nm}$ clusters appear to retain the bulk structure, and in a spherical box these clusters will be $\sim 4$ unit cells thick. We do not know the structure of the $2.5 \mathrm{~nm}$ clusters, but this size is equivalent to 2 unit cells thick. However, it is not likely that these small clusters will have any strong semblance to the bulk structure since a stack of two unit cells does not define a structure, additionally, as already noted, the optical spectrum of these clusters reveals their molecular character. In all cases we envision the clusters to be disk shaped.

\section{Isomorphous Nanoclusters}

Although we have not performed as extensive investigation of the other layered semiconductor materials such as $\mathrm{MoSe}_{2}, \mathrm{MoTe}_{2}, \mathrm{WS}_{2}, \mathrm{WSe} 2$ the optical features and size dependent bandgap shifts noted for the $\mathrm{MoS}_{2}$ also appear to hold for these materials. In addition, the synthesis and processing techniques employed for $\mathrm{MoS}_{2}$ also pertain to these systems. All layered materials investigated show the structured spectra found for $\mathrm{MoS}_{2}$. For example, WSe2 clusters show the same types of optical features as $\mathrm{MoS}_{2}$ (see Figure 29). Furthermore, a high degree of crystallinity in even $2.8 \mathrm{~nm}$ clusters is found as shown in the high resolution lattice fringe image of Figure 30.

Comparison of Figures 26 and 29 reveals the striking similarities of the optical spectra of the $\mathrm{MoS}_{2}$ and WSe 2 clusters. The absorption features for the larger WSe 2 clusters in Figure 29 are excitonic features very similar to those for the $4.5 \mathrm{~nm} \mathrm{MoS} 2$ clusters in Figure 26 and are comparable in energy. The following table compares the $\mathrm{A}$ and $\mathrm{B}$ exciton energies for the two materials in bulk and cluster forms. Specifically, for the WSe 2 clusters the A exciton peak is at $\lambda=470 \mathrm{~nm}(=2.64 \mathrm{eV})$ and the $\mathrm{B}$ exciton peak is at $\lambda=388 \mathrm{~nm}(=3.20 \mathrm{eV})$ 
yielding a splitting $\left(E_{B}-E_{A}\right)=0.56 \mathrm{eV}$. In bulk WSe2 $E_{A}=1.71 \mathrm{eV}$ and $E_{B}=2.30 \mathrm{eV}$ yielding a splitting of $0.59 \mathrm{eV}$ which is about the same as for the clusters as we find for MoS2. This A-B splitting in $\mathrm{WSe}_{2}$ is about three times larger than in $\mathrm{MoS}_{2}$ suggesting a much stronger spin-orbit coupling at the top of the valence band in the case of $\mathrm{WSe} 2$.

\begin{tabular}{|clccc|}
\hline Material & & $\mathrm{E}_{\mathrm{A}}(\mathrm{eV})$ & $\mathrm{EB}_{\mathrm{B}}(\mathrm{eV})$ & $\mathrm{EB}_{\mathrm{B}} \mathrm{E}_{\mathrm{A}}(\mathrm{eV})$ \\
\hline $\mathrm{MoS} 2$ & Bulk & 1.86 & 2.06 & 0.20 \\
& Cluster $(4.5 \mathrm{~nm})$ & 2.63 & 2.86 & 0.23 \\
\hline \multirow{2}{*}{$\mathrm{WSe}_{2}$} & Bulk & 1.71 & 2.30 & 0.59 \\
& Cluster $(\mathrm{t}=3.8 \mathrm{~min})$ & 2.64 & 3.20 & 0.56 \\
\hline
\end{tabular}

As for the quantum size confinement effect in WSe2. the A and B excitons of the clusters in Figure 29 are blue shifted by $\sim 0.90 \mathrm{eV}$ relative to the bulk. For comparison, the blue shift of these excitonic peaks for the $4.5 \mathrm{~nm} \mathrm{MoS} 2$ clusters in Figure 26 is $0.78 \mathrm{eV}$, a comparable effect. 


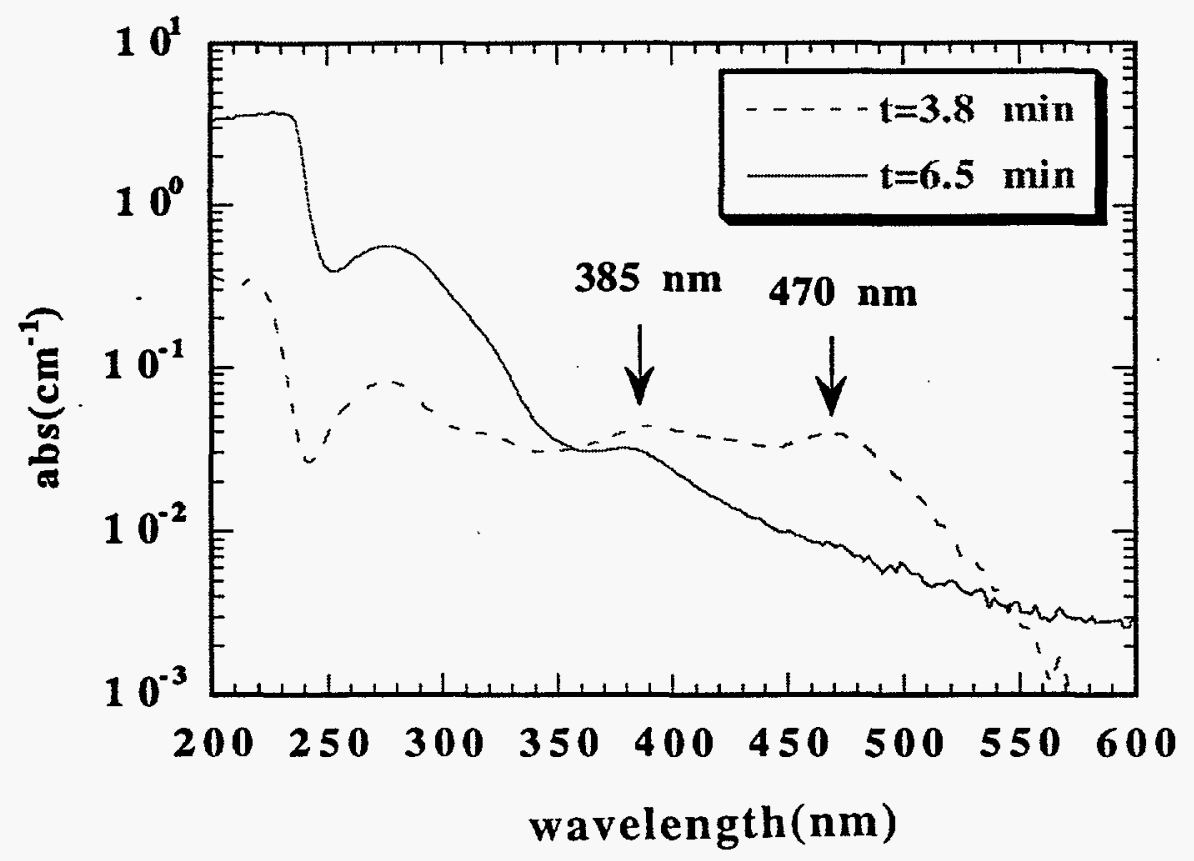

Figure 29. WSe 2 clusters processed by size exclusion chromatography on a reverse phase $120 \AA$ pore column. The larger (shorter retention time of $3.8 \mathrm{~min}$ ) clusters are yellow colored. The more strongly retained, smaller clusters $(t=6.5$ min) with a first exciton at 385 are nearly clear in color due the strong quantum confinement. 


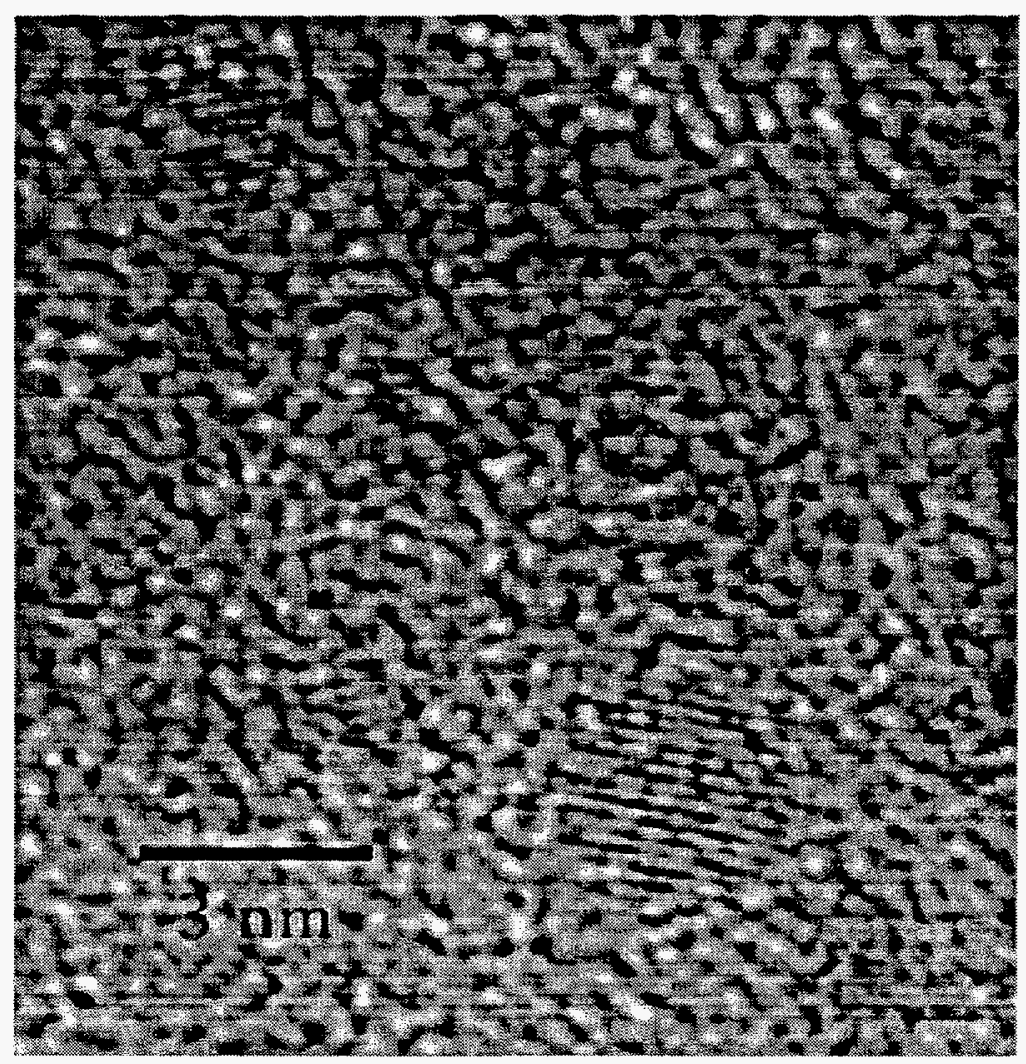

Figure 30. High resolution lattice fringe image of $2.8 \mathrm{nmWSe} 2$ cluster.

\section{E. Conclusions}

Highlights of our work on $\mathrm{MoS}_{2}$ and its isomorphs include the observation of: 1 )remarkable structure in the absorption spectra reflecting the underlying physics as well as the high quality and size monodispersity of the clusters; 2) large blue shifts in the spectral features with decreasing cluster size affording great tailorability of the bandgap; and 3) a beautiful demonstration of the crossover from band-like (solid) to molecule-like spectra as the size of the clusters becomes smaller than that of the exciton in the bulk.

Tributsch [4] was the first to suggest that the molybdenum and tungsten dichalcogenide layered compounds may have applications in solar photoelectrochemical technology. This suggestion was based on experiments using these materials in bulk form as electrodes in solar photocells which 
demonstrated high stability against photocorrosion in solution. This stability was attributed to the fact that the optical transitions responsible for the creation of the electron-hole pairs are between nonbonding metal $d$ states.[4] However, detailed band-structure calculations [17] have now shown that in these dichalcogenides the relevant $\Gamma_{4}^{-}$state at the top of the valence band is not a nonbonding metal $\mathrm{d}$ state, but rather an antibonding state between metal $\mathrm{d}_{\mathrm{z}}{ }^{2}$ and nonmetal $\mathrm{pz}$ orbitals. This antibonding character is responsible for the high stability of these materials against photocorrosion. By contrast, for materials such as $\mathrm{CdS}$ and $\mathrm{GaP}$ whose valence bands are based on p orbitals, the most stable hole states are cation-anion bonding orbitals. Excitation of electrons from these states leads to the observed photocorrosion of these materials. (see Sec. VI below).

In the present work we have demonstrated that it is possible to tailor the optical properties of these materials by controlling cluster size. This feature along with their high resistance to photocorrosion make them very attractive photocatalysts. Our future studies in this area will capitalize on these properties. 


\section{The Catalyzed Photoreduction of Methyl Orange by CdS \\ Nanoclusters}

\section{A. Motivation}

One of the main purposes of the research carried out in this program is to lay the groundwork for ultimately carrying out efficient photocatalyzed reduction and oxidation reactions. In earlier sections of this report we have presented and discussed results on a variety of semiconductor nanoclusters as well as on the modification of the surface properties of these nanoclusters which suggest that many of these materials would make excellent photocatalysts.

Our nanoclusters are grown in solution inside the micellar cages. These surfactant cages are in effect permeable capping layers, and a crucial issue is their influence on photocatalytic activity. Specifically, can reactant species get close enough to be catalyzed by the clusters? In earlier work, we have demonstrated that metal clusters grown in inverse micelles are effective in the ordinary catalysis of hydrogenation reactions and coal hydropyrolysis [3].

In this Section we shall explore this issue for a model photocatalytic system, the photoreduction of methyl orange by CdS. Although this is a model system, its use for the present purposes is instructive despite the fact that it suffers from a number of limitations. CdS has been studied extensively as a catalyst in photoreduction reactions, because its bulk bandgap $(2.4 \mathrm{eV})$ enables it to absorb a large fraction of the solar spectrum (i.e. $\lambda<516 \mathrm{~nm}$ ). In addition, the positions of its conduction band and valence band edges are well matched to several practical photoreduction and photoxidation reactions. Traditionally, bulk phase CdS powders in suspensions are studied, but unfortunately such powders exhibit severe photodegradation. We have studied nanometer sized CdS particles that do no exhibit bulk properties, but exhibit quantum size effects due to the 
spatial confinement of their electrons. The bandgap of these nanoclusters is in the near UV, and they are thus not ideal for solar photocatalysis, but are adequate for laboratory experiments using a mercury lamp. Additionally, there is no prior work on the stability of these cluster in photochemical environments.

Another issue is the large size of the methyl orange molecule which in the presence of the surfactant cage, may limit its approach to the CdS nanocluster. This indeed appears to be the case as will be discussed below. We do not expect this limitation to be very serious for photocatalyzed reactions involving small molecular species such as the dissociation of $\mathrm{H}_{2} \mathrm{O}$ and $\mathrm{H}_{2} \mathrm{~S}$. In fact, we often use $\mathrm{H}_{2} \mathrm{~S}$ in the synthesis of sulfide nanoclusters in inverse micelles where it very readily permeates the micellar cages. Similarly, $\mathrm{H}_{2} \mathrm{O}$ readily permeates these cages.

\section{B. Experimental Details}

The photoreduction of methyl orange [25] can be expressed as ( $\left.\mathrm{CH}_{3}\right)_{2} \mathrm{~N} \mathrm{C}_{6} \mathrm{H}_{4} \mathrm{~N}=\mathrm{N} \mathrm{C}_{6} \mathrm{H}_{4} \mathrm{SO}_{3}^{-}$(I)+ $2 \mathrm{H}^{+}+2 \mathrm{e}^{-}-\mathrm{CdS}_{>}$ (CH3) ${ }_{2} \mathrm{NC}_{6} \mathrm{H}_{4} \mathrm{NHNHC}_{6} \mathrm{H}_{4} \mathrm{SO}_{3}-$ (II) and is depicted schematically in Figure 31. The reaction is favored at low $\mathrm{pH}$ 's $(\mathrm{pH}=4.4)$, because the photogenerated electrons only reduce the protonated form of $I$. The role of ethylenediaminetetraacetate (EDTA) in the reaction is debated. Ideally, it is used as a sacrificial electron donor $\left(\right.$ EDTA $\left.+\mathrm{h}^{+}+--\mathrm{EDTA}^{+}\right)$to prevent anodic corrosion of the CdS particles (CdS $\left.+2 \mathrm{~h}^{+}+\ldots-\mathrm{Cd}^{2+}+\mathrm{S}\right)$. At low pH's, however, a competing reaction involving EDTA, 2EDTA ${ }^{+}+\mathrm{CdS}-\cdots--->$ $2 E D T A+C d^{2}++S$, occurs. Nevertheless, EDTA is the best sacrificial electron donor for the photoreduction of methyl orange in bulk CdS-water solutions [26]. 


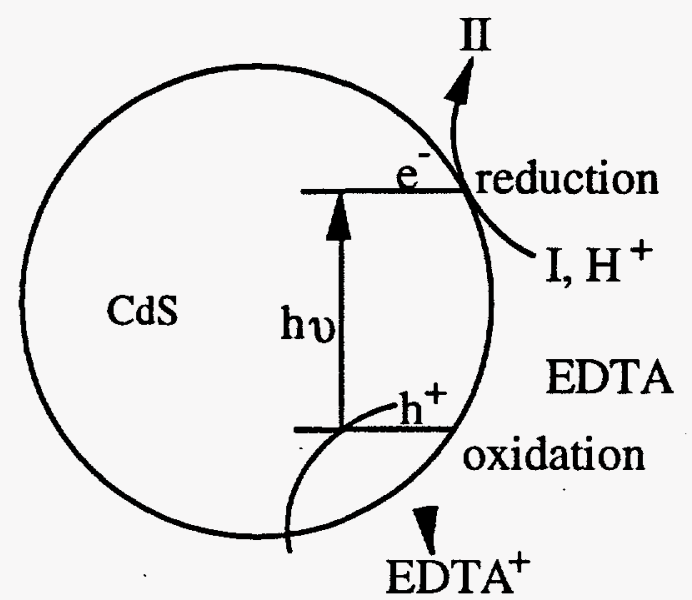

Figure 31. Schematic representation of the photoreduction of methyl orange (I) catalyzed by CdS. EDTA is depicted as a sacrificial electron donor. Anodic corrosion of the $\mathrm{CdS}, \mathrm{CdS}+2 \mathrm{~h}^{+}+\ldots--->\mathrm{Cd}^{2+}+\mathrm{S}$, occurs if EDTA does not donate sufficient electrons.

High purity methyl orange, EDTA, $\mathrm{HNO}_{3}$ and destillled and de-ionized $\mathrm{H}_{2} \mathrm{O}$ were used. Nanometer sized $\mathrm{CdS}$ was synthesized in a DTAC, octane, and hexanol inverse micelle solution as described previously in this report.

Two volume percent water adjusted to $\mathrm{pH}=4.4$ with $\mathrm{HNO}_{3}, 0.4 \mathrm{mM}$ methyl orange, and $0.01 \mathrm{M}$ EDTA are added to the CdS cluster inverse micelle solution. The water is solubilized within the interior of the inverse micelle and surrounds the $\mathrm{CdS}$ clusters. The water serves to provide an acidic environment and solubilizes the methyl orange and EDTA. The solution is mixed overnight and placed in a $25 \mathrm{ml}$ multi-port, water cooling jacketed quartz glass reactor. Flowing Ar through the solution for 15 minutes purges the reactor and solution of $\mathrm{O}_{2}$. The solution is then exposed to a 100 watt low pressure $\mathrm{Hg}$ vapor lamp while the reactor and contents are kept at $25^{\circ} \mathrm{C}$ with circulating water (Figure 32). Samples are extracted as a function of time and analyzed with a Hewlett Packard 8452A diode array spectrophotometer. The characteristic extinction peak of methyl orange $(\lambda=423 \mathrm{~nm})$ is monitored as a function of time. 

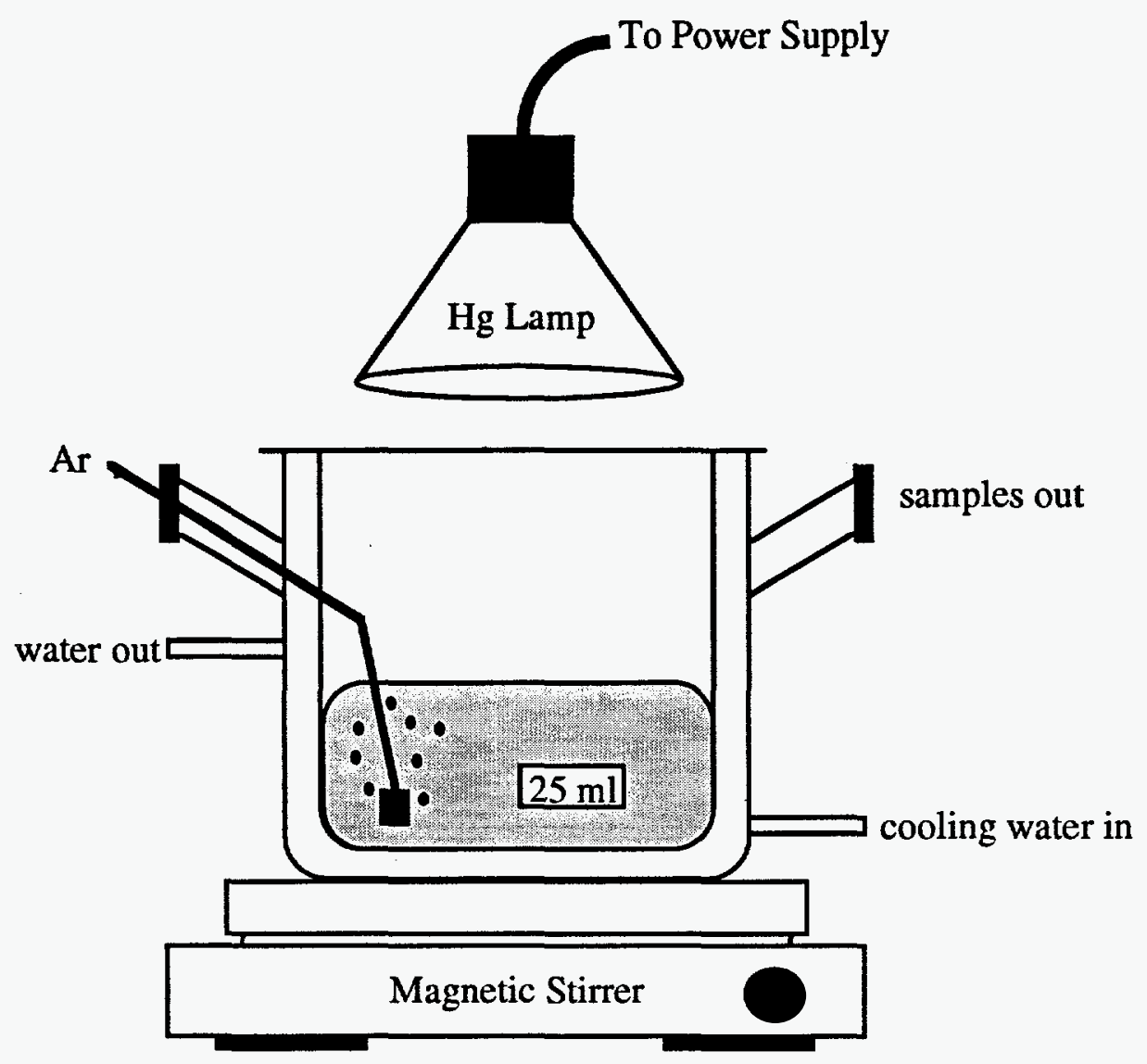

Figure 32. Schematic representation of the reactor.

\section{Results and Discussion}

Figure 33 shows the decrease in the intensity of the extinction peak of methyl orange due to the photoreduction which occurs over the course of seven hours in CdS solutions prepared by inverse micelles. In bulk powder CdS/water systems, photoreduction is complete within 30 minutes. Anodic corrosion of $\mathrm{CdS}$ nanoclusters does not explain the slow photoreduction rate in our experiment. Within 30 minutes, most of the CdS nanoclusters still exist. The most likely explanation for the slow photoreduction of methyl orange in the experiment is surface coverage of $\mathrm{CdS}$ particles with surfactant. As suspected at the outset, the surfactant layer most likely prevents diffusion of the large reactants to the particle surface. It has previously been shown that when a polymer, 
polyvinylalcohol (PVA), is used as an electron donor, the rate of photoreduction is slow. It was speculated that PVA covers the CdS particles and lessens access and reactant adsorption to the semiconductor surface. The same mechanism likely occurs in the presence of surfactant.

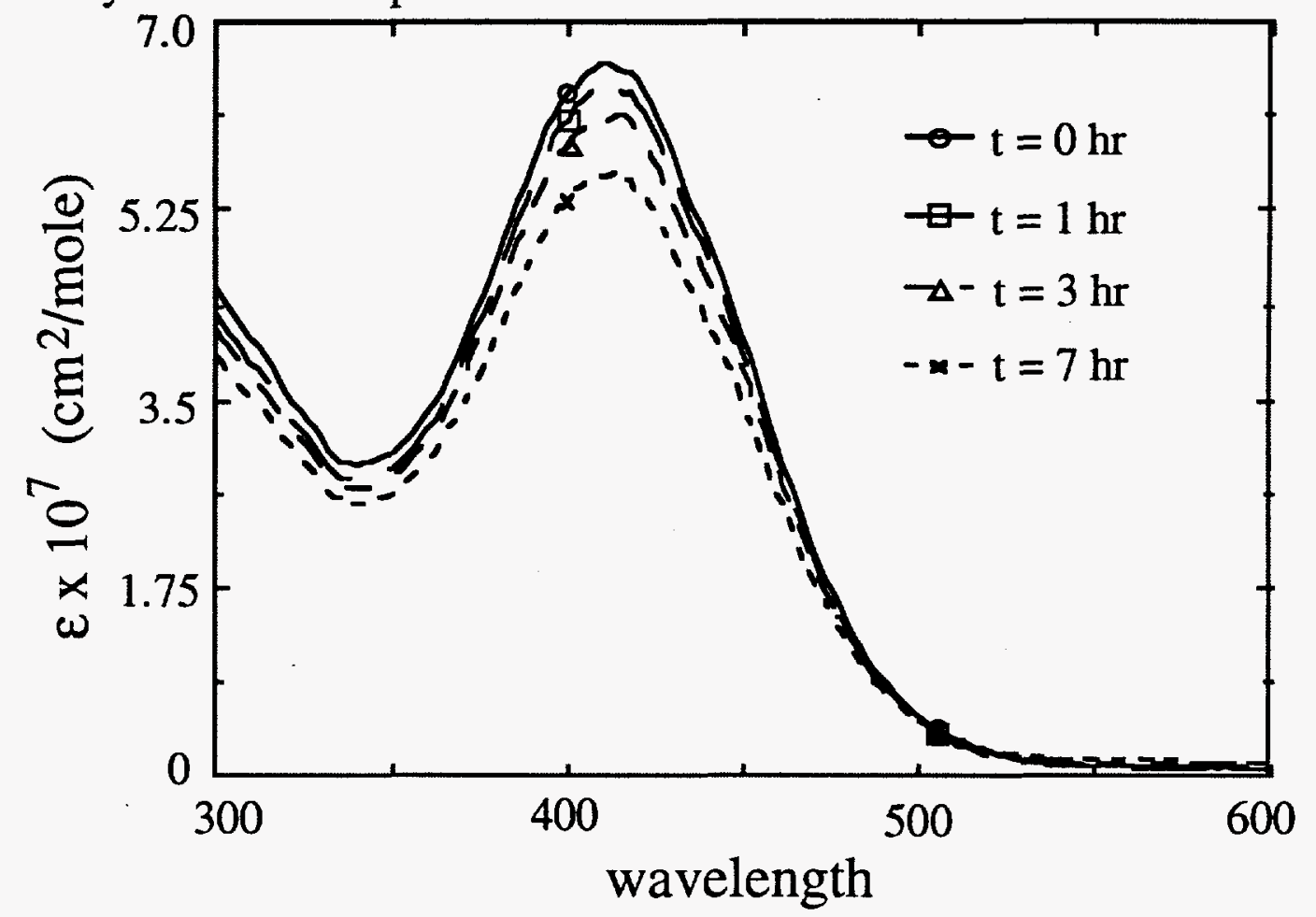

Figure 33. Extinction coefficient of methyl orange in solution as a function of time. With time, the extinction coefficient decreases, but at a much slower rate than when bulk CdS suspended in water is used as the catalyst.

CdS nanoclusters in inverse micelle solutions decompose when exposed to light (Figure 34). The extinction coefficient falls approximately $40 \%$ in seven hours, and there is a systematic blue shift with time. The decrease in absorbance most likely results from a lower concentration of $\mathrm{CdS}$ in solution. Consistent with clusters exhibiting quantum size effects, the blue shift indicates the clusters become smaller with time. The lower extinction coefficient and decreasing cluster size with exposure to light indicates a gradual decomposition of the clusters and ultimate removal of the clusters from solution. The most likely 
explanation of the cluster decomposition is anodic corrosion, $\mathrm{CdS}+2 \mathrm{~h}^{+}------>$ $\mathrm{Cd}^{2+}+\mathrm{S}$. At low pH's, EDTA may not act as an ideal electron donor and may mediate the decomposition of CdS, $2 \mathrm{EDTA}^{+}+\mathrm{CdS}----->2 \mathrm{EDTA}+\mathrm{Cd}^{2+}+\mathrm{S}$. In bulk samples of $\mathrm{CdS}$, anodic corrosion occurs, but in nanometer sized $\mathrm{CdS}$ anodic corrosion is more destructive and noticeable due to the large surface-tovolume ratio.

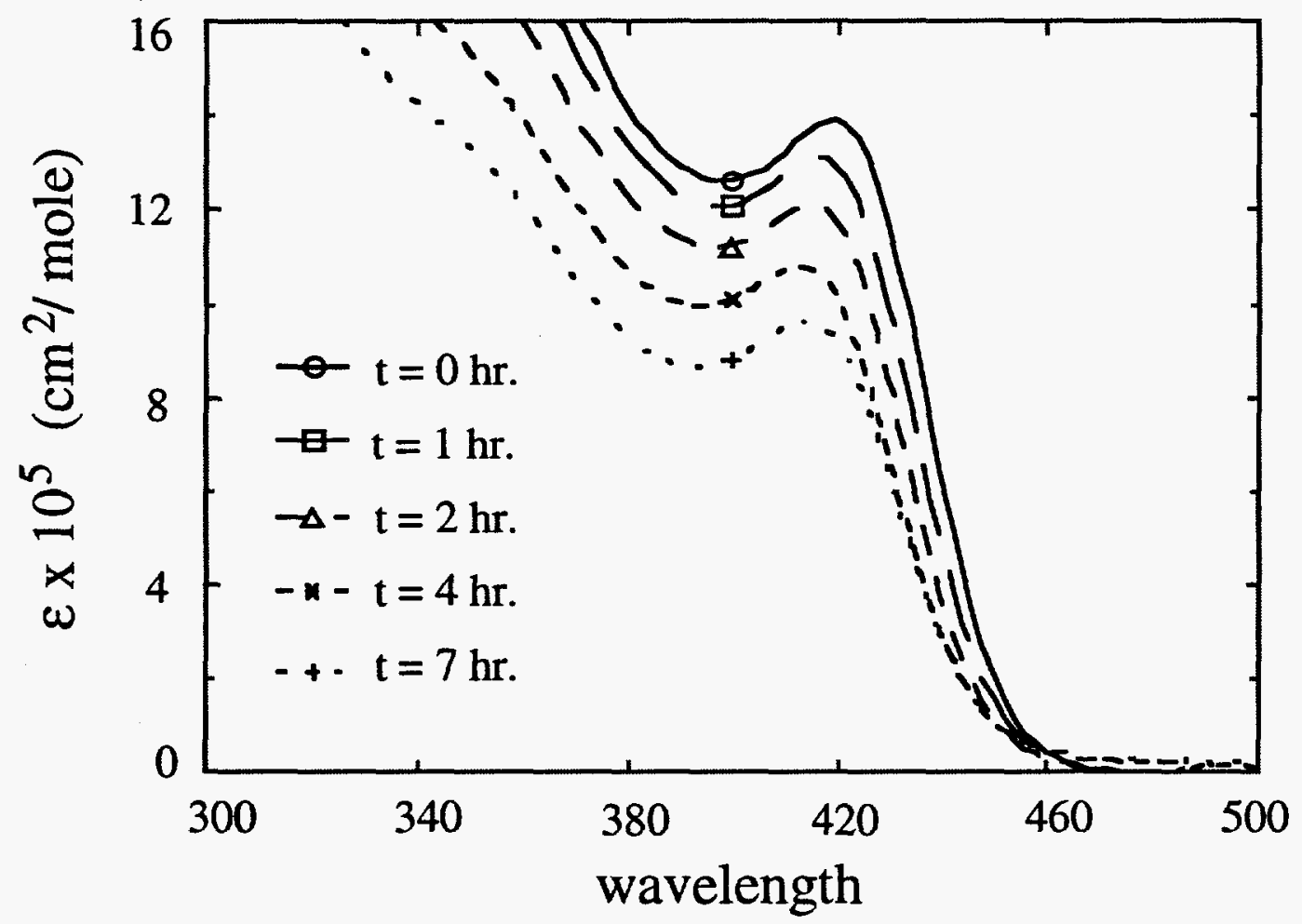

Figure 34. Extinction coefficient of nanometer sized CdS particles exposed to light as a function of time. With time, the extinction coefficient decreases and the distinctive CdS peak blue shifts. Both trends are consistent with anodic corrosion of the $\mathrm{CdS}$ particles.

\section{Concluding Remarks}

Though our photoreduction experiments with $\mathrm{CdS}$ in inverse micelles were disappointing, the other new materials produced by this program such as $\mathrm{MoS}_{2}$ and $\mathrm{WS}_{2}$ hold great promise for photoreduction reactions. Being covalent semiconductors like $\mathrm{TiO}_{2}$, they do not suffer from anodic photocorrosion. Also, 
with the new processing methods developed by this program, we can produce size controlled nanoclusters in organic media and then transfer them to water using chromatographic techniques. Thus, we can chose whichever reaction medium provides the highest efficiencies and is most suited to a given reaction. More importantly perhaps, we do not expect the micellar cages to offer a serious hindrance for the permeation of small molecule reactants such as $\mathrm{H}_{2} \mathrm{O}$ and $\mathrm{H}_{2} \mathrm{~S}$ to the cluster surface.

Finally, even materials such as $\mathrm{CdS}$ and $\mathrm{FeS}_{2}$ which are subject to photochemical degradation can be altered by the coating methods developed by this program and may yet prove useful as photocatalysts. For example, Figure 35 shows the effect on the optical properties of coating CdS with a thin layer of gold. The effect observed is very analogous to the use of dye molecules on the surface of $\mathrm{TiO}_{2}$ to extend its absorbance into the visible with a major increase in quantum efficiency. It is interesting to note the blue shift of the exciton peak due to the coating which shows the dominance of surface states in the electronic properties of these new materials. Very similar coating effects were observed in $\mathrm{FeS}_{2}$ coated with either gold or palladium as reported earlier in this SAND report. Other benefits include the increase in absorbance throughout the visible and modification of the surface carrier recombination process as discussed for coated $\mathrm{PbS}$ nanoclusters in Section III. 


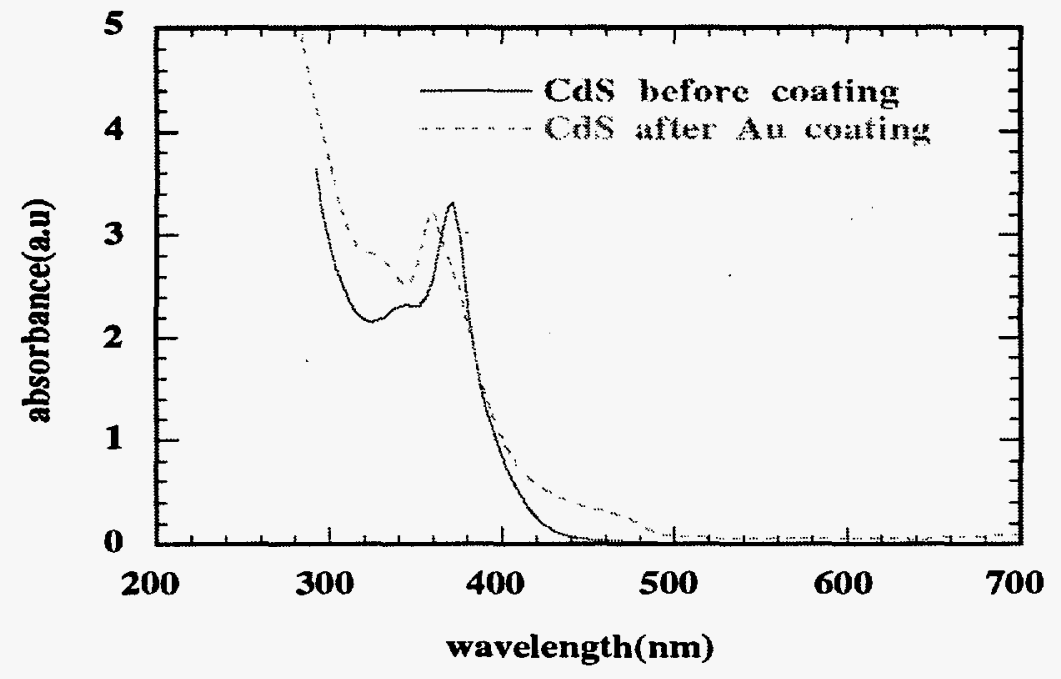

Figure 35. Effect of coating CdS with a layer of gold is to increase its absorbance far beyond the bandgap of the material while maintaining the excitonic features which allow creation of hole-electron pairs. 


\section{Future Research Directions}

The significant contribution of this LDRD to future technological application of photocatalysts is the development of several new materials, in particular the inexpensive, stable layered semi-conductors $\mathrm{MoS}_{2}$ and WS2, which in nanosize form have the right valence and conduction band potentials for many important applications such as water and $\mathrm{H}_{2} \mathrm{~S}$ splitting. Without our new approach previous extensive research on available macroscopic semiconductor powders such as $\mathrm{TiO}_{2}$ and $\mathrm{CdS}$ had shown that efficiencies for photocatalysis were unacceptably poor. Unfortunately, for fundamental reasons (e.g. fixed band-gap, photocorrosion) these efficiencies could never really be improved.

Due to this research this dead-end has now been removed, and we anticipate active research within Sandia and elsewhere on practical photocatalytic reactions using these new materials. Indeed this is an important aspect of a recently-funded BES initiative and of the BES Center for Synthesis and Processing project on nanocluster materials. We also expect these materials will prove very useful because of their inertness, lack of toxicity, and inexpensive nature (particularly $\mathrm{FeS}_{2}$ ) and will serve as a entirely new class of photooxidation catalysts for the destruction of organic wastes using sunlight (solar detoxification).

\section{Acknowledgments}

The authors would like recognize the valuable assistance of Sharon Craft in the synthesis of the materials used in this LDRD project. 


\section{References}

[1] "Modern Concepts in Surface Science and Heterogeneous Catalysis", G.A. Somorjai, J. Phys. Chem., 94, 1013-1023, (1990).

[2] Wilcoxon, J.P., DOE patent \#5,147,841, DOE control \#S 70621, entitled "Method for the Preparation of Metal Colloids in Inverse Micelles and Product preferred by the method", issued Sept. 15, 1992.

[3] "Synthesis and Characterization of Fe and FeS2 (Pyrite) Coal Liquefaction Catalysts in Inverse Micelles", A. Martino, J.P. Wilcoxon, and J. Kawola , , Energy and Fuels, $\underline{8}, 1289$, (1994).

[4] H. Tributsch, Z. Naturforsch, 32a, 972 (1977).

[5] MRS Bulletin,

[6] "Synthesis and Catalytic Properties of Metal and Semiconductor Nanoclusters", Wilcoxon J.P., Martino T., E. Klavetter, and Sylwester A.P., in Nanophase Materials, proceedings of the NATO ASI, Corfu, Greece, June 1993.

[7] Teichman, R.A., Nixon, E.R, J. Molecular Spectr. 54, 78 (1975)

[8] Barrow, R.F., Fry, P.W. and LeBargy, R. C., Proc. Phys. Soc. 81697 (1963)

[9] W. Folkerts, G. A. Sawatzky, C. Haas, R. A. deGroot and F. V. Hillebrecht, J. Phys. C: Solid State Phys. 20, 4135 (1987) and references therein.

[10] K. Sato, J. Phys. Soc. Jpn. 프, 1617 (1984).

[11] J. B. Goodenough, J. Solid State Chem. $\underline{3}, 26$ (1971).

[12] D. W. Bullett, J. Phys. C: Solid State Phys. 15, 6163 (1982).

[13] W. W. Kou and M. S. Sheehra, Phys. Rev. B $\underline{18}, 7062$ (1978).

[14] "Strong Quantum-Size Effects in a Layered Semiconductor: MoS2 Nanoclusters", J.P. Wilcoxon and G.A. Samara, , Phys Rev. B 1ㅣ, 7299, (1995).

[15] R. F. Frindt and A. D. Yoffe, Proc. Roy. Soc. A., 273, 69 (1963). 
[16] B. L. Evans and P. A. Young, Proc. Roy. Soc. A., 284, 402 (1965).

[17] R. Coehoorn, C. Haas, J. Dijkstra, C.J.F. Flipse, R.A. deGroot and A. Wold, Phys. Rev. B., 35, 6195 (1987), 35, 6203 (1987).

[18] Y. Wang, A. Suna, W. Mahler and R. Kasowski, J. Chem. Phys. 87, 7315 (1987).

[19] Al. L. Efros and A.L. Efros, Sov. Phys. Semicond. 16, 772 (1982).

[20] L.E. Brus, IEEE J. Quant. Electron QE-22, 1909 (1986).

[21]. Y. Kayanuma, Phys. Rev. B. 38, 9797 (1988), 44,13085 (1991).

[22] W. Folkerts, G. A. Sawatzky, C. Haas, R. A. deGroot and F. V.

Hillebrecht, J. Phys. C: Solid State Phys. 20, 4135 (1987) and references therein.

[23] See A. D. Yoffe, Adv. in Phys. 42, 173 (1993) for a recent review and references therein.

[24] J.R. White and A.J. Bard, J. Phys. Chem., 1985, 89, 1947.

[25] A. Mills and G. Williams, J. Chem. Soc., Faraday Trans. 1, 1987, 83, 2647.

[26] A.J. Bard and M.S. Wrighton, J. Electrochem. Soc., 1977, 124, 1706.

[27] G.T. Brown and J.R. Darwent, J. Chem. Soc., Faraday Trans. 1, 1984, 80, 1631. 


\section{Appendix}

List of Publications and Presentations

\section{Publications}

D.E. Bliss, J.P. Wilcoxon, and G. Samara, Optical Properties of Lead Sulfide Nanoclusters: Effects of Size, Stoichiometry and Surface Alloying, Proceedings of Symposium F, meeting of the MRS Society, Boston, MA., (1994 ).

J.P. Wilcoxon, G. Samara, and P. Newcomer, Optical Features of Nanosize Iron and Molybdenum Sulfide Clusters, Proceedings of Symposium F, meeting of the MRS Society, Boston, MA., (1994).

J.P. Wilcoxon and G.A. Samara, Strong Quantum-Size Effects in a Layered Semiconductor: MoS $_{2}$ Nanoclusters, Physical Rev B 51,7299 (1995).

Venturini E.L., Wilcoxon J.P., and Newcomer P.P., Magnetic Properties of Nanosize Iron Clusters, Symposium V, Proceedings of the Spring Meeting of the Materials Research Soc., San Francisco, CA. (1994).

A. Martino, J.P. Wilcoxon, and J. Kawola, "Synthesis and Characterization of Fe and $\mathrm{FeS}_{2}$ (Pyrite) Coal Liquefaction Catalysts in Inverse Micelles", Energy and Fuels, $\underline{8}$, 1289, (1994).

Wilcoxon J.P., Martino T., E. Klavetter, and Sylwester A.P., Synthesis and Catalytic Properties of Metal and Semiconductor Nanoclusters, in Nanophase Materials, ed. G. Hadjipanaysis and D. Siegel, Kluwer Acad. Pub., 770-780, 1994.

\section{Presentations:}

J.P. Wilcoxon, G. Samara, and P. Newcomer, Optical Features of Nanosize Iron and Molybdenum Sulfide Clusters, Symposium F, 1994 meeting of the MRS Society, Boston, MA.

D.E. Bliss, J.P. Wilcoxon, and G. Samara, Optical Properties of Lead Sulfide Nanoclusters: Seffects of Size, Stoichiometry and Surface Alloying, Symposium F, 1994 meeting of the MRS Society, Boston, MA.

J.P. Wilcoxon, G. Samara, and P. Newcomer, Optical and Catalytic Properties of Nanosize Metal Sulfide Clusters, 1994 meeting of the American Physical Society, March 22 1994, Pittsburgh, PA

Wilcoxon J.P., Martino T., E. Klavetter, and Sylwester A.P., Synthesis and Catalytic Properties of Metal and Semiconductor Nanoclusters, in Nanophase Materials, NATO ASI meeting, Corfu, Greece, June 1993. (invited) 
J.P. Wilcoxon, G. Samara, and P. Newcomer, Strong Quantum-Size Effects in a Layered Semiconductor: MoS2 Nanoclusters, 1993 meeting of the American Physical Society, March 22, 1993, Seattle, WA.

A. Martino, J. Wilcoxon, A.P. Sylwester, and J.S. Kawola, Synthesis and Characterization of Fe and FeS2 (pyrite) Catalysts Particles in Inverse Micelles, Proceedings of the 205th National American Chemical Soc. meeting, Miami, (1993).

List of Invention Disclosures: None

List of patents: None

List of copyrights: None

Employee recruitment: Post doc. David Bliss hired.

Student Involvement: None

Follow On Work: New BES funded initiative on Nanoclusters for Energy Applications funded. 
Distribution:

1 MS-1436 C. E. Meyers, 1007

1 MS-1427 P. L. Mattern, 1100

1 MS-1421 D. Bliss, 1152

1 MS-1421 J. E. Martin, 1152

1 MS-1421 P. P. Newcomer, 1152

10 MS-1421 G. A. Samara, 1152

5 MS-1421 J. P. Wilcoxon, 1152

1 MS-1134 B. K. Damkroger, 1833

1 MS-1134 R. L. Williamson, 1833

1 MS-0710 G. A. Carlson, 6211

1 MS-0710 A. Martino, 6211

1 MS-9018 Central Tech Files, 8523-2

5 MS-0899 Technical Library, 13414

1 MS-0619 Print Media, 12615

2 MS-0100 Document Processing, 7613-2

44 DOE/OSTI 